\title{
Examining and strengthening the role of patent and proprietary medicine vendors in the provision of injectable contraception in Nigeria
}

\author{
Salisu Mohammed Ishaku \\ Population Council \\ Kayode Afolabi \\ Sara Chace Dwyer \\ Population Council \\ Faizah Okunade \\ Chiamaka Uzomba
}

See next page for additional authors

Follow this and additional works at: https://knowledgecommons.popcouncil.org/departments_sbsr-rh How does access to this work benefit you? Let us know!

\section{Recommended Citation}

Ishaku, Salisu Mohammed, Kayode Afolabi, Sara Chace Dwyer, Faizah Okunade, Chiamaka Uzomba, Adedamola Adebayo, Elizabeth Tobey, and Aparna Jain. 2018. "Examining and strengthening the role of patent and proprietary medicine vendors in the provision of injectable contraception in Nigeria," Research report. Washington, DC: Population Council, The Evidence Project. 


\section{Authors}

Salisu Mohammed Ishaku, Kayode Afolabi, Sara Chace Dwyer, Faizah Okunade, Chiamaka Uzomba, Adedamola Adebayo, Elizabeth Tobey, and Aparna Jain 


\section{Examining and Strengthening the Role of Patent and Proprietary Medicine Vendors in the Provision of Injectable Contraception in Nigeria}

Salisu Ishaku, Population Council, Nigeria

Kayode Afolabi, Federal Ministry of Health, Nigeria

Sara Chace Dwyer, Population Council, Washington D.C.

Faizah Okunade, Population Council, Nigeria

Chiamaka Uzomba, Population Council, Nigeria

Adedamola Adebayo, Population Council, Nigeria

Elizabeth Tobey, Population Council, Washington D.C.

Aparna Jain, Population Council, Washington D.C.

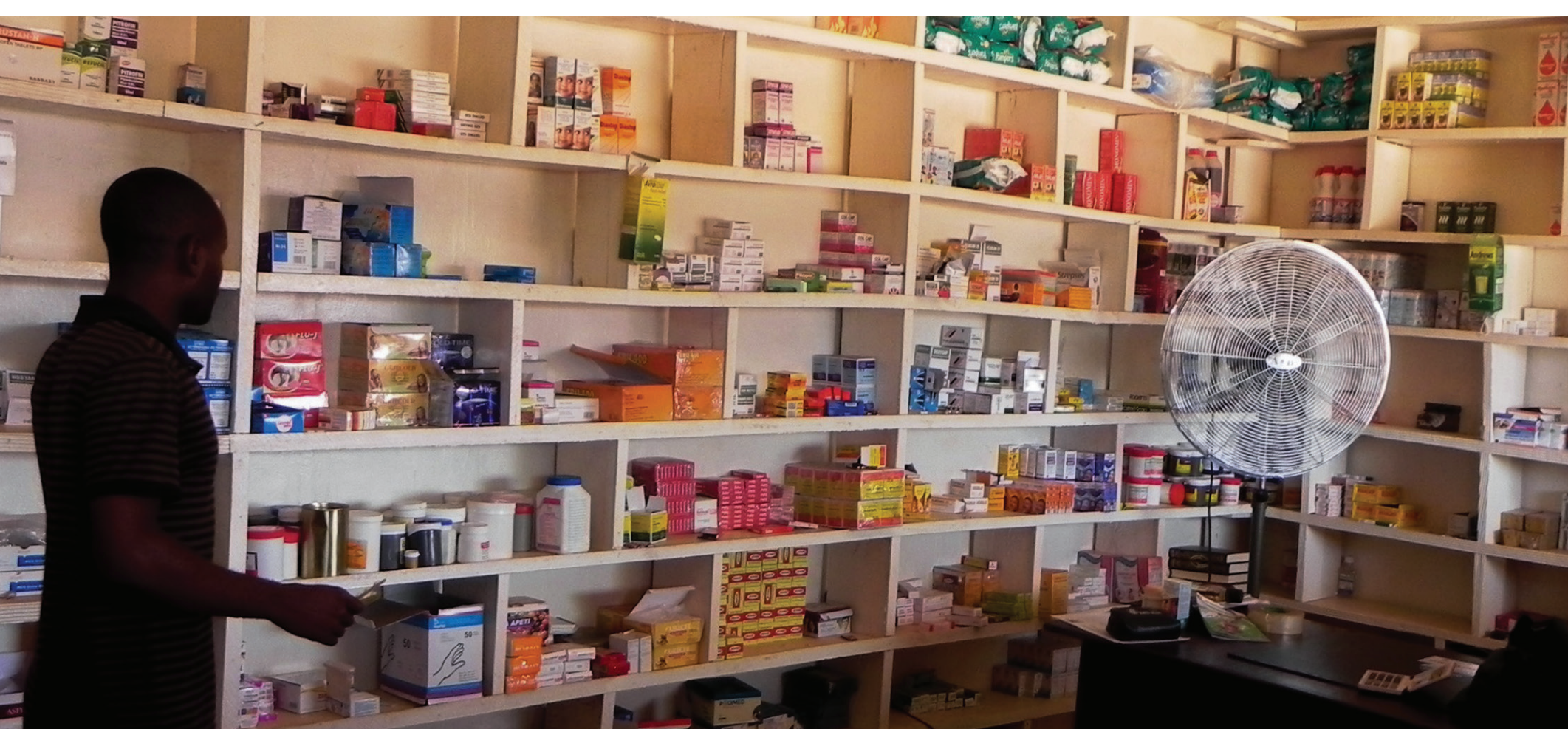




\section{The Evidence Project}

Population Council

4301 Connecticut Avenue, NW, Suite 280

Washington, DC 20008 USA

tel +1 2022379400

(3) USAID

The Evidence Project is made possible by the generous support of the American people through the United States Agency for International Development (USAID) under the terms of cooperative agreement no. AID-OAA-A-13-00087. The contents of this document are the sole responsibility of the Evidence Project and Population Council and do not necessarily reflect the views of USAID or the United States Government.

The Evidence Project uses implementation science-the strategic generation, translation, and

use of evidence-to strengthen and scale up family planning and reproductive health programs to reduce unintended pregnancies worldwide. The Evidence Project is led by the Population Council in partnership with the Population Reference Bureau.

Published in October 2018.

Suggested citation: Ishaku, Salisu, Kayode Afolabi, Sara Chace Dwyer, Faizah Okunade, Chiamaka Uzomba, Adedamola Adebayo, Elizabeth Tobey, and Aparna Jain. 2018. "Examining and Strengthening the Role of Patent and Proprietary Medicine Vendors in the Provision of Injectable Contraception in Nigeria," Research Report. Washington, DC: Population Council, The Evidence Project.

(C) 2018. The Population Council, Inc. 


\section{Examining and Strengthening the Role of Patent and Proprietary Medicine Vendors in the Provision of Injectable Contraception in Nigeria}

Salisu Ishaku, Director Reproductive Health Programs, Population Council, Nigeria

Kayode Afolabi, Federal Ministry of Health, Nigeria

Sara Chace Dwyer, Staff Associate, Population Council, Washington D.C.

Faizah Okunade, Program Officer, Population Council, Nigeria

Chiamaka Uzomba, Project Officer, Population Council, Nigeria

Adedamola Adebayo, Project Officer, Population Council, Nigeria

Elizabeth Tobey, Research Specialist, Population Council, Washington D.C.

Aparna Jain, Associate II, Population Council, Washington D.C.

OCTOBER 2018 


\section{Table of Contents}

Acknowledgments............................................................................

List of Acronyms .............................................................................

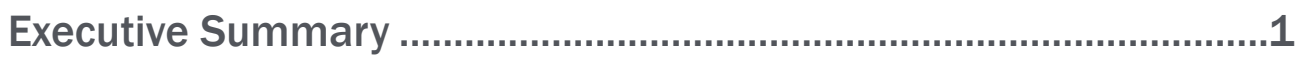

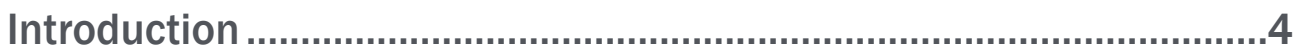

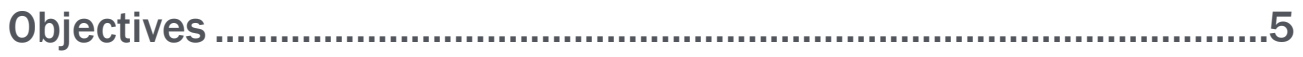

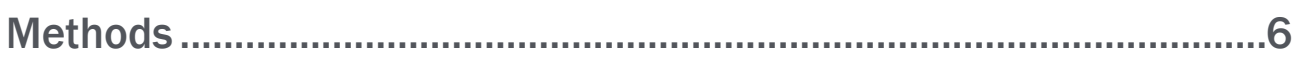

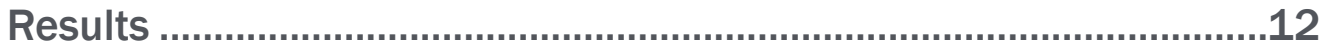

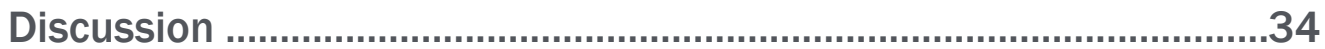

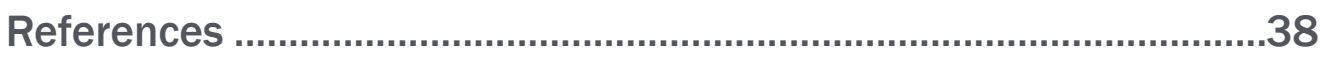




\section{List of Tables}

Table 1. PPMV characteristics at pretest survey ........................................................................ 12

Table 2. PPMV knowledge of contraceptive methods at pretest survey.........................................13

Table 3. Comparison of PPMV injectable knowledge among those who had and had not previously worked in a health facility, post-test survey ................................................... 17

Table 4. Comparison of PPMV injectable knowledge among those who had and had not previously worked in a health facility, 9-month follow-up survey.

Table 5. Knowledge comparisons of needle safety practices at pretest and post-test surveys .....25

Table 6. Knowledge comparisons of needle safety practices at post-test and 9-month surveys ...28

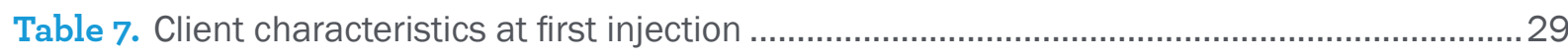

Table 8. Reasons for stopping injectable method at second and third interview ...........................33

\section{List of Figures}

Figure 1. Contraceptive methods sold by PPMVs at pretest survey ........................................... 13

Figure 2. Injectable contraceptive services provided by PPMVs at pretest survey .....................13

Figure 3. Type of contraceptive methods sold by PPMVs at pretest survey................................14

Figure 4. Comparison of PPMV Demonstration of key DMPA-IM administration steps at post-test and 1-month monitoring visit......

Figure 5. Comparison of PPMV demonstration of key DMPA-IM administration steps at 1-month monitoring visit and 6-month monitoring visit

Figure 6. Comparison of PPMV demonstration of key DMPA-SC administration steps at post-test and 1-month monitoring visit......

Figure 7. Comparison of PPMV demonstration of key DMPA-SC administration steps at 1-month and 6-month monitoring visits.....

Figure 8. Comparison of monitoring team observations of PPMVs' practices at 1-month and 6-month monitoring visits

Figure 9. Comparison of PPMV demonstration of key DMPA-IM administration steps among those who had and had not previously worked in a health facility, post-test.... 20

Figure 10. Comparison of PPMV demonstration of key DMPA-SC administration steps among those who had and had not previously worked in a health facility, post-test....20

Figure 11. Comparison of PPMV demonstration of key DMPA-IM administration steps among those who had and had not previously worked in a health facility, 6-month monitoring visit.

Figure 12. Comparison of PPMV demonstration of key DMPA-SC administration steps among those who had and had not previously worked in a health facility, 6-month monitoring visit. 
Figure 13. Knowledge comparisons of seven common side effects of the injectable at pretest and post-test surveys

Figure 14. Knowledge comparisons of 11 exclusionary health conditions of the injectable at pretest and post-test surveys

Figure 15. Knowledge comparisons of when a woman can start the injectable at pretest and post-test surveys

Figure 16. Knowledge comparisons of post-administration instructions at pretest and post-test surveys

Figure 17. Knowledge comparisons of key injectable method characteristics at pretest and post-test surveys

Figure 18. Knowledge comparisons of seven common side effects of the injectable at post-test and 9-month surveys

Figure 19. Knowledge comparisons of 11 exclusionary health conditions of the injectable at post-test and 9-month surveys

Figure 20. Knowledge comparisons of when a woman can start the injectable at the post-test and 9-month surveys

Figure 21. Knowledge comparisons of post-administration instructions at the post-test and 9-month surveys

Figure 22. Knowledge comparisons of key injectable method characteristics at post-test and 9-month surveys

Figure 23. Outcome of client's first visit to a trained PPMV for injectable services

Figure 24. Clients' reasons for choosing PPMVs for injectable contraceptive services at first injection by a trained PPMV.

Figure 25. Reporting of side effects to PPMVs or other providers, among continuing injectable clients who experienced side effects in the preceding three months

Figure 26. Quality of care received at first injection by trained PPMVs.

Figure 27. Quality of care received by continuing clients at follow-up injections by trained PPMVs

Figure 28. Other aspects of continuing clients' visits to trained PPMVs for injectable contraceptive services. 


\section{Acknowledgments}

The study was conducted in collaboration with the Federal Ministry of Health of Nigeria and Ministries of Health in Bauchi, Cross River, Ebonyi, Kaduna, Nasarawa and Oyo States. We also acknowledge the role of the Pharmacists Council of Nigeria, which was an integral partner during the conceptualization, implementation and monitoring in Bauchi, Cross River, Ebonyi and Kaduna States and the National Association of Patent and Proprietary Medicine Vendors for its collaboration in recruiting and monitoring PPMVs. We acknowledge all stakeholders (government, private and non-profit agencies) and individuals-Professor Ademola Ajuwon from the University of Ibadan, Joseph Monehin and Alaa El-Bashir from the United States Agency for International Development (USAID), and Mihira Karra, Magwa Baker and Erika Martin from USAID Washington, D.C.who provided valuable inputs in the design and/or implementation of the study. We also acknowledge Osasuyi Dirisu for her oversight of the data management in Abuja, Laura Reichenbach for her guidance in the study implementation and for her review of an earlier version of the report, and Robin Johnson and Anneka Van Scoyoc of the Evidence Project team for editing and formatting this report. The successful completion of the study would not have been possible without the dedication of the Patent and Proprietary Medicine Vendors in the participating states. We further acknowledge all the study clients, who gave their time and provided valuable information during the study. 


\section{List of Acronyms}

BCS+ Balanced Counseling Strategy Plus

DMPA Depot medroxyprogesterone acetate

FMoH Federal Ministry of Health

FP Family Planning

ID Identification

LGA Local Government Areas

NAPPMED National Association of Patent and Proprietary Medicine Dealers

NDHS National Demographic Health Survey

NET-EN Norethisterone enanthate

MEC Medical Eligibility Criteria

MICS Multiple Indicator Cluster Survey

PCN Pharmacist Council of Nigeria

PM Patent Medicine

PPMV Patent and Proprietary Medicine Vendor

RH Reproductive Health

SPSS Statistical Package for Social Sciences

USAID United States Agency for International Development 


\section{Executive Summary}

Nigeria is committed to achieving a modern contraceptive prevalence rate of 27 percent by 2020; yet current estimates show only 11 percent of women age 15-49 years old use modern contraceptive methods. Among both public and private family planning (FP) service providers, Patent and Proprietary Medicine Vendors (PPMV) are the most popular source for voluntary contraceptive services. According to Nigeria's 2013 National Demographic Health Survey, 38 percent of all contraceptive users and 13 percent of injectable users received their method from a PPMV.

The Evidence Project, led by the Population Council with support from the U.S. Agency for International Development, collaborated with the Federal Ministry of Health $(\mathrm{FMoH})$, state ministries of health, and professional associations to conduct an implementation science study in Bauchi, Cross River, Ebonyi, Kaduna, Nasarawa and Oyo States representing each of Nigeria's six geo-political zones. The study objectives were to:

- Assess the feasibility of PPMVs in the provision, specifically the administration, of voluntary injectable contraceptives ${ }^{1}$; and

- Explore injectable contraceptive users' experiences and quality of care received when accessing contraceptive services from PPMVs.

PPMVs received a 1-week training on FP counseling, sale, referral and administration of all progestin-only injectable contraceptives. Trainings in Nasarawa and Oyo were held in November 2015, and in Bauchi, Cross River, Ebonyi and Kaduna in May and June of 2017. Learnings from Nasarawa and Oyo were applied to the implementation process in Bauchi, Cross River, Ebonyi and Kaduna, including an observation checklist that was added to the monitoring visits one and six months after the training. Monitoring teams comprised of national, state and local-level ministry officials, National Association of Patent and Proprietary Medicine Dealers (NAPPMED) and Pharmacy Council of Nigeria (PCN) representatives visited PPMVs two-to-three times over nine months.

Trained data collectors conducted face-to-face interviews with PPMVs before, after, and nine months following the training. Injectable contraceptive clients were interviewed within five days after receiving an injection from a trained PPMV and were followed over nine months by phone. Written informed consent was obtained from all respondents participating in the study. The research protocol received ethical approval from the Population Council's Institutional Review Board, National Health Research Ethic Committee, the Ethical Committee at University of Ibadan and ethics committees based at state ministries of health in Bauchi, Cross River, Ebonyi and Kaduna.

Almost all PPMVs were providing some form of injectable contraceptive services (counseling, sale, referral or administration) and 49 percent had administered an injectable in their shop before the training. Forty percent of clients had visited a study-enrolled PPMV for injectable services before the trainings. Key study findings are summarized below:

PPMVs can administer injectable contraceptives safely

- PPMVs demonstrated competency in nine key steps for safe administration of intramuscular depot

1 DMPA-IM (Depo-Provera), NET-EN (Noristerat) and DMPA-SC (Sayana Press) 
medroxyprogesterone acetate (DMPA-IM) and nine key steps for safe administration of subcutaneous DMPA (DMPA-SC) using models immediately and six months after the training in front of a Master Trainer in Bauchi, Cross River, Ebonyi and Kaduna.

- Nearly all PPMVs (98\%) were observed having a sharps disposal box in use in their shop by the monitoring teams in Bauchi, Cross River, Ebonyi and Kaduna.

There was no difference in knowledge and skills between PPMVs with and without previous health facility experience

- PPMVs without prior health facility experience had similar or higher levels of knowledge to those with health facility experience on:

- 44 of the 50 injectable contraceptive characteristics at the post-training survey

- 46 of the 50 injectable contraceptive characteristics at the 9-month follow-up survey

- PPMVs with no health facility experience demonstrated similar levels of competency in almost all the steps required for safe administration of DMPA-IM and DMPA-SC to those with health facility experience.

\section{PPMVs' knowledge of injectable contraceptive characteristics was higher after the training and PPMVs retained this knowledge nine months later}

- PPMVs had significantly higher knowledge on 47 of 50 injectable contraceptive indicators ${ }^{2}$ post-training.

- PPMV retained or improved knowledge for 42 of the 50 indicators at the 9-month follow-up survey. Knowledge specifically increased for 13 of those 42 indicators.

- Despite overall knowledge gains across most indicators, knowledge on some indicators like side effects and exclusionary health conditions remained low at the 9-month follow-up survey. Non-prompted questions were used to assess PPMVs knowledge. These questions may not accurately reflect PPMVs knowledge as clients reported receiving key information from PPMVs, specifically side effects, and $86 \%$ of PPMVs reported using either the MEC wheel, BCS cards and or DMPA screen checklist, when counseling their clients.

\section{Clients reported receiving quality FP counseling and were satisfied with injectable services received from PPMVs}

- Over 80 percent of clients reported receiving information on 9 quality of care indicators ${ }^{3}$ at their first injection visit from a trained PPMV. At their second and third injection visits, most clients continued to report quality counseling.

- Over 97 percent of clients reported that they would return to the PPMV for their next injection and 100 percent would recommend that PPMV to a friend for injectable contraceptive services.

2 The 50 indicators are described in the Results section starting on page 20 and include: side effects (7), exclusionary health conditions (11), when a woman can start the injectable (6), instructions for clients after the injection (6), method-specific characteristics (9), how to handle needles safely (5), how to avoid infection from needlestick injury (3), and what to do if there is a needlestick injury (3)

3 The 9 quality of care indicators include: (1) being asked the reason for your visit; (2) told about other FP methods; (3) asked about previous use of the injectable (4) asked about reasons for choosing the injectable (5) told when to return for the next injection (6) told what to do if you experiences a problem with the injectable; (7) told about potential side effects (8) told about potential actions to resolve problems with the side effect; and (9) reported feeling comfortable asking questions. 


\section{Clients who discontinued the injectable contraceptive did so for reasons unrelated to PPMV}

service quality

- Of the 22 percent of clients (66/294) in Bauchi, Cross River, Ebonyi and Kaduna who reported discontinuing the injectable contraceptive method or stopping injectable services from PPMVs, the top three reasons were: didn't have time to go back (21\%); wanted to become pregnant $(20 \%)$, and side effects (18\%).

Clients reported a variety of reasons for seeking injectable contraceptive services from PPMVs. Many do so because it is more convenient. Policy change that would allow PPMVs to offer injectable administration could improve access to FP counseling and injectable contraceptives for more women throughout Nigeria. If policy were to be changed, the following considerations could assist in scale-up:

- The curriculum used in this study was based on existing, validated materials. Most PPMVs strongly agreed that the curriculum used in this study improved their knowledge and skills to provide FP counseling and voluntary injectable contraceptive services. Using existing materials can help save time when training PPMVs in injectable contraceptive service provision.

- Job aids that are designed to help providers adhere to counseling and screening guidelines are acceptable to PPMVs. Most PPMVs used the job aids that were provided in the study; job aids can help alleviate the burden of memorizing long lists of information.

- Better linkages between the PPMVs and the public sector are needed to ensure effective referrals for any contraceptive method and proper disposal of sharps.

- PPMVs require monitoring support by the state and local governments. Additional support would be needed to ensure PPMVs do not experience harassment from law officials. This potential for harassment can prohibit PPMVs from keeping up-to-date records of FP counseling, and sale and administration of injectable contraceptives. 


\section{Introduction}

With a population of approximately 214 million people, Nigeria is Africa's most populous nation (Population Reference Bureau, 2018). Modern contraceptive methods remain underutilized in Nigeria (only $11 \%$ of married women of reproductive age use modern contraceptives) [MICS, 2017] despite efforts to expand access to FP over the past two decades. Approximately 40 percent of modern contraceptive users use an injectable contraceptive, 21 percent use oral contraceptives, 13 percent use an implant, 10 percent use male condoms and 16 percent use various other methods (MICS, 2017). Among both public and private sources, PPMVs are the most popular source for modern contraceptives (NPC and ICF, 2014) for many reasons including (a) widespread availability throughout Nigeria, (b) consistent drug stocks, (c) extended operational hours including weekends, (d) personable interactions, and (e) no separate fees for consultations (Brugha 2002; Adetunji 1991). Nigerian pharmacy laws prohibit PPMVs from selling and administering injectable contraceptives due to a lack of formal training in injectable contraceptive services (NPC, 2008 \& 2003). Even with this restriction, 13 percent of injectable contraceptive users received an injectable contraceptive from a PPMV (NPC and ICF, 2014).

The FMoH recognizes the important role that PPMVs can play in the delivery of modern contraceptive services and recommends that PPMVs should motivate, counsel, and refer clients for contraceptive services, supply non-prescription contraceptives, and resupply oral contraceptive pills (FMoH, 2009). Although several studies have explored the role of PPMVs in offering contraceptive services broadly (Oye-Adeniran et al, 2006; Ajayi, 2009), few have specifically assessed the contribution of PPMVs in delivering injectable contraceptives, the implementation considerations, and their clients' experiences receiving these services (Ajuwon et al. 2013). For these reasons, the Evidence Project, led by the Population Council and funded by the United States Agency for International Development (USAID), collaborated with the FMoH and state ministries of health to conduct an implementation science study to assess the feasibility and acceptability of PPMVs' provision of injectable contraceptive services.

This report summarizes the results of the implementation science study and identifies programmatic implications for PPMVs to safely deliver injectable contraceptive services, including the mentorship and support that would be needed if PPMVs were legally allowed to administer injectable contraceptives. Results from this study will contribute to task shifting ${ }^{4}$ policy in Nigeria with the potential to improve women's access to contraceptive services offered by PPMVs.

4 Task shifting is the shifting of specific tasks or services that have traditionally been done by specialized health workers to those with less training (WHO, 2007) 


\section{Objectives}

The goal of this study was to improve access of voluntary family planning services for women and couples in Nigeria. The study objectives were to:

- Assess the feasibility of PPMVs in the provision, specifically the administration of DMPA-IM (DepoProvera), NET-EN (Noristerat), and DMPA-SC (Sayana Press); and

- Explore the experiences of injectable contraceptive users and the quality of care they receive when accessing injectable contraceptive services from PPMVs. 


\section{Methods}

\section{Study design}

The study was implemented in Bauchi, Cross River, Ebonyi, Kaduna, Nasarawa and Oyo states. PPMVs enrolled in the study participated in a five-day training covering FP counseling and the provision of progestin-only injectable contraceptive services (including counseling, sale, referral and administration), and were routinely monitored after the training. USAID Washington funded implementation in Nasarawa and Oyo states. The USAID Mission in Nigeria provided additional funding to expand the study to Bauchi, Cross River, Ebonyi, and Kaduna so that the overall study included one state from each geo-political zone. Ministry officials, Master Trainers, non-governmental organizations (NGOs), professional associations, and the Population Council modified the intervention and the study design for Bauchi, Cross River, Ebonyi and Kaduna by reviewing results and implementation experiences from Oyo and Nasarawa. The modifications included (a) adjusting the PPMV data collection and monitoring time points, (b) adding questions to the PPMV and client survey tools, (c) updating the training curriculum, and (d) providing PPMVs with job aids.

The overall study design remained the same despite these modifications. In all six states, the Evidence Project collected data from PPMVs and their injectable contraceptive clients over a 9-month period.

\section{Study population and recruitment}

The study took place in six of the 36 states in Nigeria: Bauchi, Cross River, Ebonyi, Kaduna, Nasarawa and Oyo. The states were selected in collaboration with the FMoH and the USAID Mission in Nigeria to represent each of the six geopolitical zones of Nigeria. In each state, four Local Government Areas (LGAs), (two urban and two rural) were randomly selected by simple balloting for a total of twenty-four. The categorization of rural or urban LGAs was obtained from state government websites. The number and selection of LGAs were determined in collaboration with the respective states' ministries of health.

\section{Patent and proprietary medicine vendors}

PPMVs are frontline service providers who operate in Nigeria's informal health sector. In Nigeria, a PPMV is defined by the Pharmacy Law as someone who is licensed by the State Ministry of Health to sell pre-packaged, over the counter medications $(\mathrm{FMoH}, 1946)$. The Pharmacy Law requires that applicants be at least 21 years old and submit the names of two references to obtain a license. By custom, PPMVs are expected to have completed a minimum of primary school education (Liu, 2014). Most PPMVs learned to sell and dispense medicine through apprenticeships with licensed PPMVs.

In Nasarawa and Oyo, announcements were made during NAPPMED meetings in each of the study LGAs. NAPPMED is the main professional association of PPMVs in Nigeria. The announcement included the study's goal and methodology, and the training PPMVs would receive if enrolled. The local NAPPMED chapter nominated PPMV members using three criteria:

1. Interested and willing to participate

2. Able to read and write in English, and speak English and either Yoruba or Hausa

3. Committed to attend all training sessions 
NAPPMED sent a shortlist of candidates to the research team. PPMVs were randomly selected from that list to participate in the study. Selected PPMVs were sent formal invitations with information on the dates and location for the training.

In Bauchi, Cross River, Ebonyi and Kaduna, PPMVs were primarily recruited in collaboration with PCN, which is the regulatory body for PPMVs in Nigeria. PCN was more actively engaged in the additional four states due to its key role in overseeing PPMV services. Each PCN state officer nominated PPMVs based on the following criteria:

- Licensed with PCN

- Interested and willing to participate

- Able to read and write in English

- Committed to attend all training sessions

NAPPMED assisted with PPMV recruitment in LGAs where PPMV licensure with PCN was low. As in Nasarawa and Oyo, PPMVs were randomly selected from the shortlist of candidates identified by PCN. Selected candidates were contacted and invited to trainings at the state level. PPMVs were retained in the study if they demonstrated competence in administering DMPA-IM and DMPA-SC injections on dummy models. A Master Trainer used an observation checklist to evaluate PPMV skills.

\section{Injectable contraceptive clients}

For a 3-month period after the training, participating PPMVs referred eligible clients to be interviewed for the study. The referral period was extended to four months in Bauchi, Cross River, Ebonyi and Kaduna to reach the desired sample size. Eligible clients were those who were administered a progestin-only injectable contraceptive from a trained PPMV. PPMVs informed clients about the study and requested permission to share the client's name and phone number with the study team for follow-up. If a client agreed to participate, a trained data collector called the client within five days of her visit to the PPMV. At the time of the telephone interview, the interviewer asked a set of questions to ensure that the respondent was aware of the study and then read the informed consent statement (see section on Ethical Considerations) before proceeding with the questionnaire. Any client who came to a PPMV outside of the 3- to 4-month referral window was not enrolled in the study.

\section{Data collection}

\section{Data collectors training}

Four data collectors and one supervisor were recruited in each state to conduct PPMV and client interviews. Data collectors and supervisors were trained in four-day data collection training. Content included:

- an overview of the study and its goals

- a description of PPMVs and their role in the health care system

- a discussion of the importance of research ethics, including privacy and confidentiality

- the PPMVs' role in ensuring confidentiality, privacy, and anonymity

- informed consent and the consent forms

- thorough review of interview questions and their purpose 
- the data collection timeline

- data management

The training combined traditional lecture with adult learning methodologies including role play and mock interviews with clients and PPMVs. Mock interviews were conducted with non-participating PPMVs identified by the local NAPPMED chapter, and volunteers acted as mock clients. The supervisors received additional information on (a) their role as a supervisor, (b) the data management process, (c) how to identify data collection issues, and (d) to how to seek guidance from the research team to resolve issues.

\section{Data collection}

Nasarawa and Oyo

Data collection for the PPMVs began in November 2015. Data collected from PPMVs included (a) a pre-test survey and (b) 1-, 3-and 9-month follow-up surveys. The pretest survey was administered before the training and included demographic questions and knowledge questions on injectable contraceptives (e.g. frequency and administration location, counseling on side effects and method effectiveness, eligibility criteria, supplies, and proper storage and disposal of drugs). Follow-up surveys were conducted in person at the PPMV's shop one, three and nine months after the training. The follow-up surveys were identical at each time point and included questions on the type of injectable services provided, and the knowledge questions in the pretest survey.

Initial telephone interviews with clients were conducted between December 2015 and February 2016. The first interview included questions on (a) client demographics, (b) previous FP and injectable use, (c) type of services received, (d) experience and satisfaction with PPMV services, and e) satisfaction with the injectable method. The same survey, minus the demographic questions, was administered after the respondents' expected second and third injection. A 2-week window after the expected injection date was used to interview clients for the follow-up surveys. Clients who were no longer using the injectable or did not receive the injectable from a trained PPMV were not interviewed but were contacted again at the time of the next follow-up interview.

\section{Baucbi, Cross River, Ebonyi and Kaduna}

Data collection for PPMVs in Bauchi, Cross River, Ebonyi and Kaduna began in May 2017. Minor modifications were made to data collection process based on the experience in Nasarawa and Oyo. A post-test training survey was included to compare knowledge immediately before and after the training. The follow-up surveys were conducted only three and nine months after the training to avoid duplication with the monitoring visits (see section on Monitoring and Supervision).

The pretest and follow-up surveys were almost identical to those used in Oyo and Nasarawa, but included additional questions related to PPMV knowledge of NET-EN and their stock of other FP methods. Data collectors administered the post-test on the last day of the training. The post-test included the same set of questions as the pretest survey, excluding the demographic and store practice questions.

Follow-up surveys were conducted in person at the PPMV's shop three and nine months after the training. The surveys included questions on the type of injectable services provided since the training and the knowledge questions from the pre-test survey.

Initial telephone interviews with clients were conducted between May 2017 and September 2017. The first interview and follow-up surveys were similar to those used in Nasarawa and Oyo except the follow-up surveys 
included a subset of questions for clients who stopped using the injectable or stopped receiving injectable services from PPMVs. Several questions on quality were also added to the client surveys. Based on the experience in Nasarawa and Oyo, the interview window for follow-up surveys was extended from two to four weeks after scheduled injection date.

\section{Data management and analysis}

\section{Data management}

Data collection supervisors checked survey data for completeness and accuracy. Completed questionnaires were sent to the Population Council office in Abuja for review by the Program Officer. All completed surveys were stored in locked files at the Population Council office and access to the surveys was strictly limited to the research team. Only the Program Officer had access to respondent names, study identification numbers, and the instruments. Completed questionnaires were entered into Statistical Package for Social Sciences (SPSS) software and analyzed using Stata 14 software.

\section{Data analysis}

Descriptive statistics and bivariate analyses were conducted. Chi-square tests were used to determine significant associations over time and between PPMVs with and without previous health facility experience. T-tests were used to determine significance differences in the mean knowledge scores between the initial phase (Nasarawa and Oyo) and the expanded phase (Bauchi, Cross River, Ebonyi and Kaduna).

\section{Ethical considerations}

\section{Ethical review}

The research protocol, including the data collection tools and informed consent forms, received ethical approval from the Population Council's Institutional Review Board, and the Ethical Committee, College of Medicine at the University of Ibadan. Ethical approval was also received in each state. The FMoH approved the study. All data collection activities involving human subjects began only after full approvals were received from relevant ethical review boards.

\section{Informed consent}

Structured, written informed consent forms were used during in-person interviews with PPMVs and over the phone with clients. The consent form provided respondents with (a) information on the study objectives, (b) methodology, (c) measures to protect their privacy, confidentiality and anonymity, and (d) who to contact for additional information or if there was a problem. Before the start of each survey, a trained data collector read the informed consent form and explained its contents. PPMVs were given a copy to read and keep. A data collector asked each PPMV for his/her written consent and clients for their verbal consent over the phone. Informed consent was received by all respondents at the start of each survey.

\section{Confidentiality}

Each respondent received a unique identification (ID) number during the first interview. This unique ID code was recorded on the questionnaires and no other personal identifying information was recorded on the questionnaires. Documents containing names of participants, including the informed consent forms, were kept physically separate from the questionnaires in a locked cabinet at the Population Council office in Abuja, Nigeria. 


\section{Description of the Intervention}

\section{Stakeholder engagement}

The Evidence Project collaborated with stakeholders throughout the life of the study including in the design, implementation, and results interpretation phases. For example, the original protocol only included training PPMVs to counsel, sell and refer clients for injectable services. To ensure the study's results would provide policymakers and program implementers with the evidence needed, the Population Council in coordination with the FMoH presented the original protocol to the National Reproductive Health Technical Working Group. At this meeting, Technical Working Group members highlighted the need for evidence on PPMV administration of injectable contraceptives. As a result, the protocol and intervention were expanded to include administration. Stakeholder engagement continued in the monitoring visits where national, state and local-level ministry officials were members of the monitoring team (see section on Monitoring and Supervision).

The Evidence Project, federal, state and local ministries of health, NGOs and professional associations reviewed the training curriculum used in Nasarawa and Oyo alongside results from these two states. The curriculum was revised to improve outcomes for certain topics. Examples of revisions made to the curriculum and training include:

- reduce lecture time and increase hands-on practice and small group activities

- provide a clearer definition of common side effects versus adverse events

- simplify language used in the screening checklist for injectable users

The revised curriculum was used in Bauchi, Cross River, Ebonyi and Kaduna. PPMVs in these states were asked a series of question at the 9-month survey to understand their perceptions of the training $(n=194)$. One hundred percent agreed with the following two statements, 1) "the training improved my knowledge and skills to provide injectable contraceptives" and 2) "the training improved my knowledge and skills in FP counseling" (data not shown). When asked how easy or difficult they found the training, 72 percent said the training was easy to very easy and 26 percent said it was moderate. Only 2 percent of PPMVs found the training to be difficult or very difficult.

Overall, knowledge outcomes were not higher in the additional four states compared to knowledge outcomes in Oyo and Nasarawa. Knowledge of injectable contraceptive side effects, however, was higher in Bauchi, Cross River, Ebonyi and Kaduna. The mean number of side effects named by PPMVs in Nasarawa and Oyo was 3.2 compared to 3.8 in the additional four states at the 9-month survey (p-value 0.01). In addition, fewer PPMVs in Bauchi, Cross River, Ebonyi and Kaduna incorrectly reported side effects as adverse and severe adverse events during the follow-up surveys and monitoring visits. For example, PPMVs in Nasarawa and Oyo frequently reported common side effects (e.g. irregular bleeding, weight gain, spotting) as adverse events. This revealed that many PPMVs confused normal side effects with the adverse events that should be reported. In Phase II, only two PPMVs incorrectly reported side effects as adverse event.

\section{PPMV training}

Trainings were held in November 2015 in Nasarawa and Oyo, and in May and June 2017 in Bauchi, Cross River, Ebonyi and Kaduna. PPMVs were trained to provide FP counseling and progestin-only injectable contraceptive services (counsel, sell, refer and administer). Master Trainers who were certified to lead FP trainings in 
Nigeria facilitated the trainings. The curriculum was based on a set of training materials developed by PATH on DMPA-SC ${ }^{5}$ and FHI 360 on DMPA injections for public providers. ${ }^{6}$ The training included adult learning methodologies such as role play, group discussion, and hands-on practice. PPMVs were trained to counsel and screen clients using the World Health Organization's Medical Eligibility Criteria (MEC) Wheel, the Population Council's Balanced Counseling Strategy Plus (BCS+) and FHI 360's Checklist for Screening Clients Who Want to Initiate DMPA (or NET-EN).

Based on learnings from Nasarawa and Oyo, PPMVs were given job aids at the end of the trainings in Bauchi, Cross River, Ebonyi and Kaduna to ensure they could properly screen and counsel clients. Each PPMV was given a WHO MEC wheel, a copy of the BCS+ cards, and the FHI 360 DMPA screening checklist. During the stakeholders meeting to review the curriculum, the FHI 360 DMPA screening checklist was simplified to ensure that PPMVs with all levels of education could correctly screen clients interested in using the injectable. PPMVs in the Bauchi, Cross River, Ebonyi, and Kaduna were given job aids during the training and were asked about their use of job aids during the 9-month survey. The majority of PPMVs (86\%) reported using the job aids with some to all of their clients when providing FP services (data not shown). Among those who reported using job aids with their clients ( $\mathrm{n}=168), 78$ percent reported using the WHO MEC Wheel, 70 percent reported using BCS+, and 46 percent reported using the FHI 360 screening checklist.

\section{Monitoring and supervision}

A monitoring team comprised of the research staff, a Master Trainer, and key stakeholders visited PPMVs in their shops after the training. The purpose of the monitoring visits was to: (1) observe the quality of injectable services offered by PPMVs, especially drug storage and disposal of sharps; (2) provide support and mentorship to PPMVs; and (3) provide stakeholders an opportunity to see the study's progress, discuss implementation challenges and solutions with PPMVs, and see what would be needed to broaden this program in their state. The monitoring team used a form that included questions on the implementation challenges faced by PPMVs and questions similar to those on knowledge and service provision in the follow-up survey.

In Nasarawa and Oyo, each PPMV was visited one, three and nine months after the training. The monitoring team consisted of members from the research team, a Master Trainer, and federal, state and local ministry of health staff, and a NAPPMED representative. Based on the monitoring team's observations, and overall results from Nasarawa and Oyo, some changes were made to the monitoring visits conducted in Bauchi, Cross River, Ebonyi and Kaduna. First, a PCN representative in the project state was included in the monitoring team. This was to ensure that PCN as the regulatory body for PPMVs, could observe PPMVs progress and provide guidance on implementation challenges. Second, PPMVs were monitored one and six months after the training. This reduced the total number of monitoring visits from three to two to avoid duplication with other data collection activities. Third, PPMVs were asked to role play administering DMPA-IM and DMPA-SC in front of the Master Trainer. The Master Trainer assessed PPMVs' skills using the observation checklist used at the posttest. Fourth, the monitors answered questions related to their observations of the PPMV's shop to understand whether PPMVs were properly storing and disposing sharps and keeping records of injectable clients.

5 https://www.path.org/resources/dmpa-sc-training-materials/

6 https://www.fhi360.org/sites/default/files/media/documents/checklist-dmpa-english.pdf 


\section{Results}

A total of 388 PPMVs participated in the five-day training. Seven PPMVs were dropped after the training because they either did not demonstrate competence in injectable administration by the end of the training or did not participate in all the sessions. This brought the total sample size to 381. In Nasarawa and Oyo, four PPMVs were unavailable for the post-test survey, which took place one month after the training. ${ }^{7}$ By the 9-month survey, 38 PPMVs were either dropped from the study or were unavailable at the time of data collection. This brought the total sample for the 9-month survey to 343. Analyses were conducted cross-sectionally and included data from all PPMVs interviewed at each respective survey time-point.

\section{PPMV characteristics and family planning services provided before the training}

Table 1 shows the characteristics of PPMVs enrolled in the study at the pretest survey. Enrolled PPMVs were a diverse group of service providers. The majority $(72 \%)$ were male, 67 percent were between 30 and 49 years of age, and 92 percent had at least a secondary education. Twenty-eight percent had previously worked in a health facility and only 21 percent had been working as a PPMV for less than five years. Two-thirds of PPMVs (74\%) reported that their shops were open seven days a week.

At the pretest survey, PPMVs were asked the FP methods that they knew, the types of FP methods they sold, and types of injectable contraceptive services that they provide. Table 2 shows PPMVs' unprompted responses to FP knowledge. The majority of PPMVs knew about the male condom (93\%), the pill (90\%), and the injectable $(86 \%)$. Only 28 percent knew of emergency contraceptives and less than a quarter could name the IUD $(23 \%)$ the implant (24\%), or the vaginal ring (24\%). Fewer

7 In Nasarawa and Oyo, the post-test survey was conducted one month after the training. For the purposes of the analysis, these data were combined with post-test data from Bauchi, Cross River, Ebonyi and Kaduna.

\section{TABLE 1. PPMV CHARACTERISTICS AT PRETEST SURVEY} $(\mathrm{N}=\mathbf{3 8 1})$

\begin{tabular}{|c|c|}
\hline & Percentage \\
\hline \multicolumn{2}{|l|}{ Sex } \\
\hline Male & 71.9 \\
\hline Female & 28.1 \\
\hline \multicolumn{2}{|l|}{ Age } \\
\hline $17-29$ & 19.7 \\
\hline $30-39$ & 41.7 \\
\hline $40-49$ & 25.5 \\
\hline $50+$ & 13.1 \\
\hline \multicolumn{2}{|l|}{ Highest level of education } \\
\hline Primary school & 8.4 \\
\hline Secondary school (WASC/GCE) & 44.4 \\
\hline Two years of post-secondary (OND/NCE) & 25.7 \\
\hline College degree (HND/1st degree) & 16.3 \\
\hline Post graduate degree/other & 5.2 \\
\hline \multicolumn{2}{|l|}{ Years working as a PPMV } \\
\hline Less than 5 & 20.5 \\
\hline $5-9$ & 20.7 \\
\hline $10-14$ & 19.9 \\
\hline 15 or more & 38.9 \\
\hline \multicolumn{2}{|l|}{ Have previously worked in a health facility } \\
\hline Yes & 27.8 \\
\hline No & 72.2 \\
\hline \multicolumn{2}{|l|}{ Number of days PM shop is open } \\
\hline 7 & 73.8 \\
\hline 6 & 24.9 \\
\hline 5 or fewer & 1.3 \\
\hline \multicolumn{2}{|l|}{ State } \\
\hline Bauchi & 15.0 \\
\hline Cross River & 13.1 \\
\hline Ebonyi & 15.7 \\
\hline Kaduna & 20.0 \\
\hline Nasarawa & 20.0 \\
\hline Oyo & 21.0 \\
\hline
\end{tabular}


TABLE 2. PPMV KNOWLEDGE OF CONTRACEPTIVE METHODS AT PRETEST SURVEY ( $\mathrm{N}=381)$

\begin{tabular}{l|c}
\hline \multicolumn{1}{c}{ Contraceptive Method } & Percentage \\
\hline Tubal ligation & 5.1 \\
Vasectomy & 9.9 \\
Pill & 90.3 \\
IUD/Loop & 22.8 \\
Injectables & 86.1 \\
Implant (n=225) ${ }^{\dagger}$ & 23.6 \\
Vaginal ring & 23.6 \\
Male condom & 93.2 \\
Female condom & 61.7 \\
Emergency contraception & 28.1 \\
Diaphragm & 7.6 \\
Spermicide & $\vdots .5$ \\
Cycle beads & 41.7 \\
Withdrawal & 11.8 \\
Rhythm method & 6.8 \\
\hline
\end{tabular}

†Only asked of PPMVs in Bauchi, Cross River, Ebonyi and Kaduna could name a permanent method: tubal ligation (5\%) and vasectomy $(10 \%)$. PPMVs were asked which FP methods they sold in their shops (Figure 1). PPMVs' knowledge of FP methods seemed to correspond with the type of FP methods that they sold. The three most commonly sold FP methods were the pill (87\%), male condoms (75\%) and injectable contraceptives (59\%).

Almost all PPMVs $(92 \%)$ provided at least one injectable contraceptive service at the pre-test survey. Results in Figure 2 show that approximately 60 percent reported they counsel clients on injectable use, sell injectable methods or refer clients to the nearest health facility for injectable services. Forty-seven percent said they administer injectable contraceptives in their shop. Figure 3 shows the type of injectable contraceptive methods sold by PPMVs in their shops. PPMVs primarily sold Depo-Provera (58\%) and Noristerat (45\%). Less than two percent reported selling Cyclofem, Mesigyna, or Sayana Press before the training.

\section{FIGURE 1. CONTRACEPTIVE METHODS SOLD BY PPMVS AT PRETEST SURVEY $(\mathrm{N}=381)$}

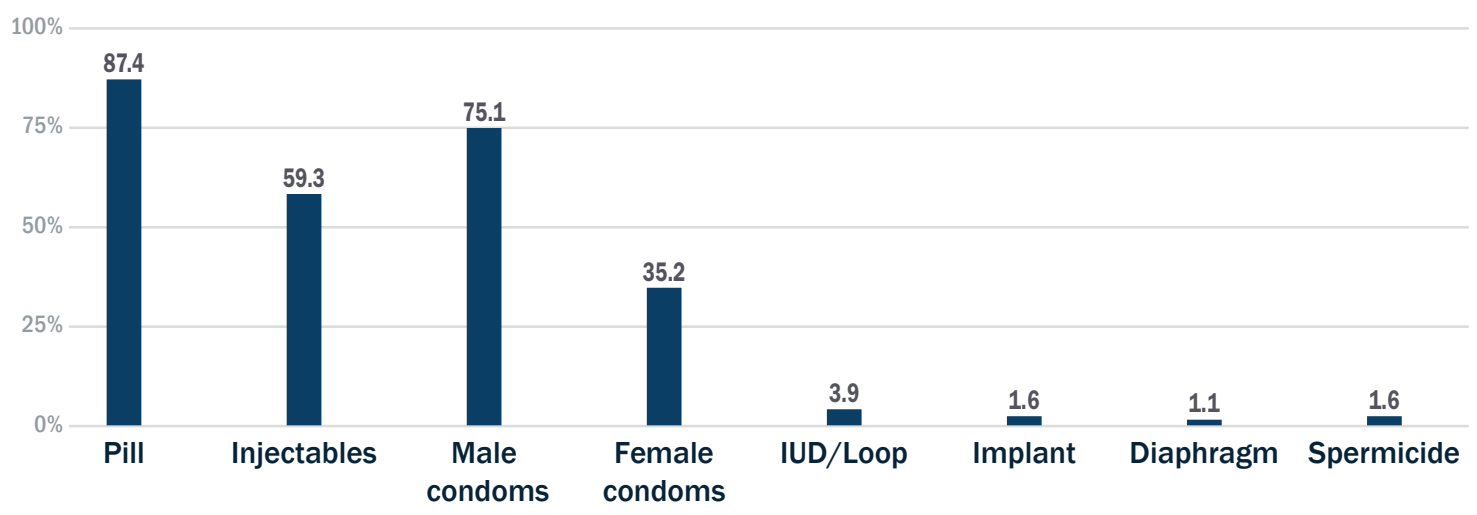

FIGURE 2. INJECTABLE CONTRACEPTIVE SERVICES PROVIDED BY PPMVS AT PRETEST SURVEY $(\mathbf{N}=381)$

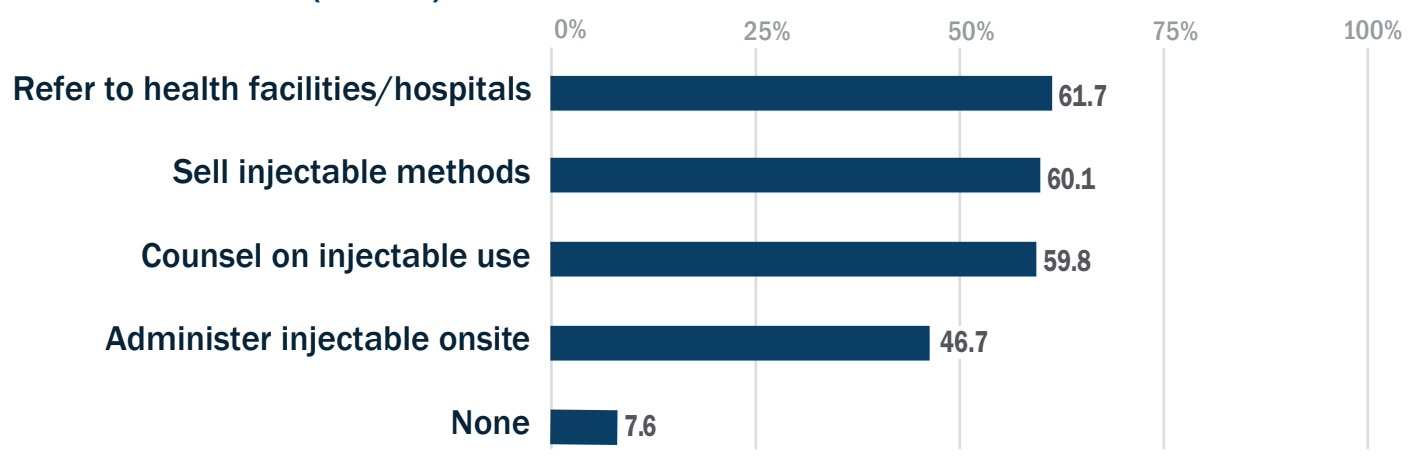




\section{FIGURE 3. TYPE OF CONTRACEPTIVE METHODS SOLD BY PPMVS AT PRETEST SURVEY $(\mathrm{N}=\mathbf{3 8 1})$}

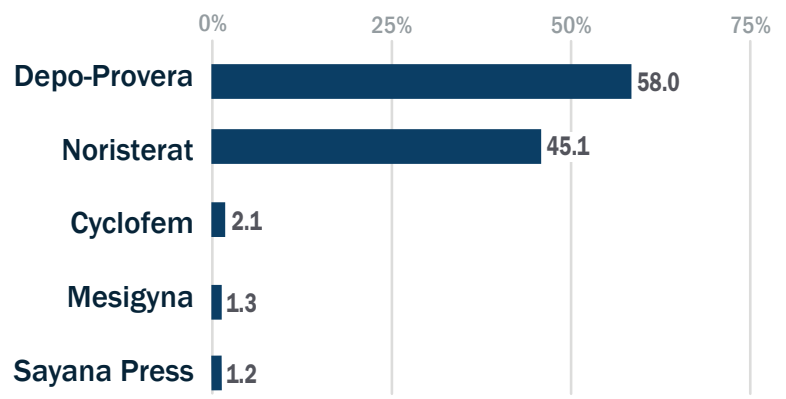

\section{PPMVs can administer} injectable contraceptives safely

The observation checklist for PPMV administration of DMPA-IM and DMPA-SC was conducted in Bauchi, Cross River, Ebonyi and Kaduna immediately after the training and during the 1 - and 6-month monitoring visits. The majority of PPMVs from these states demonstrated key steps for safe

administration of injectable contraceptives, even six months after the training. Figure 4 shows the proportion of PPMVs who demonstrated the key DMPA-IM administration steps during the post-test and 1-month monitoring visit. Results show that at least 75 percent of PPMVs demonstrated nine key steps for safe administration of DMPA-IM after the training ( $\mathrm{n}=225)$. There were slight decreases for all but one of the steps at the 1-month monitoring visit ( $\mathrm{n}=196)$. Only for one DMPA-IM step, pierce top of vial with sterile needle and fill syringe, was there no difference observed from post-test to the 1-month visit (94\% compared to 95\%). Even though most of these decreases were statistically significant, at least 50 percent of PPMVs demonstrated each of the nine steps at the 1-month monitoring visit.

PPMVs' skills improved between the 1- and 6-month monitoring visits. Figure 5 shows changes in the proportion of PPMVs who demonstrated the key DMPA-IM administration steps at the 1-and 6-month monitoring visits. A greater number of PPMVs demonstrated six of the nine key DMPA-IM steps compared to the 1-month observation. For example, the proportion of PPMVs who checked for the expiration date increased from 74 percent at the 1 -month monitoring visit to 87 percent at the 6 -month monitoring visit ( $\mathrm{p}$-value $\leq 0.01$ ). Only the DMPA-IM step aspirate needle remained low at the 6-month monitoring visit (56\%). Eighty-five percent of PPMVs in Bauchi, Cross River, Ebonyi and Kaduna, however, named this step when asked during the 9-month follow-up survey (data not shown).

\section{FIGURE 4. COMPARISON OF PPMV DEMONSTRATION OF KEY DMPA-IM ADMINISTRATION STEPS AT POST-TEST AND 1-MONTH MONITORING VISIT ${ }^{\dagger}$}

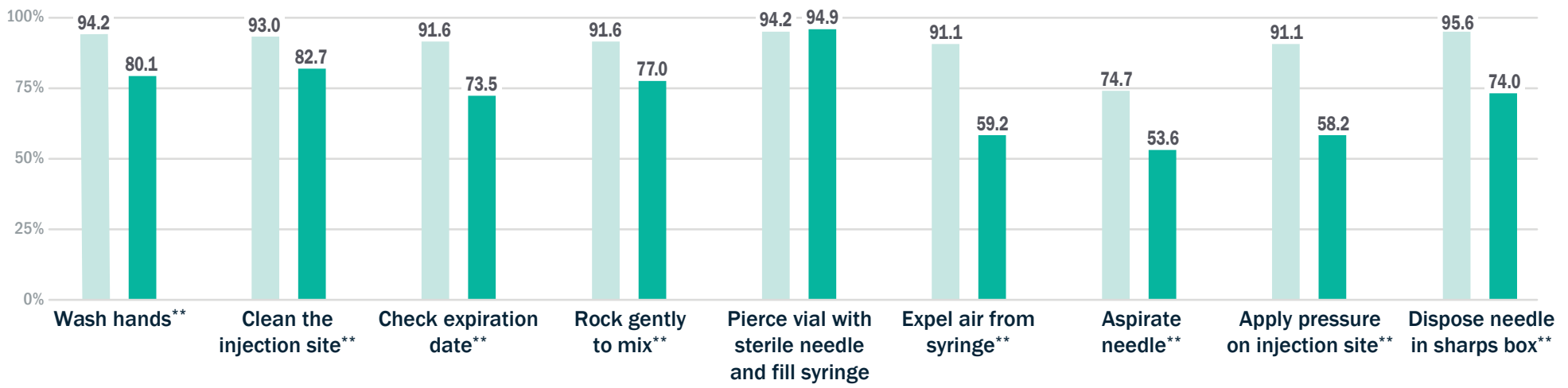

†Observations used for PPMVs in Bauchi, Cross River, Ebonyi and Kaduna

${ }^{*} p$-value $\leq 0.05 ;{ }^{* *} p$-value $\leq 0.01$ Post-test $(n=225) \quad$ 1-month monitoring visit $(n=196)$ 
FIGURE 5. COMPARISON OF PPMV DEMONSTRATION OF KEY DMPA-IM ADMINISTRATION STEPS AT 1-MONTH MONITORING VISIT (N=196) AND 6-MONTH MONITORING VISIT'

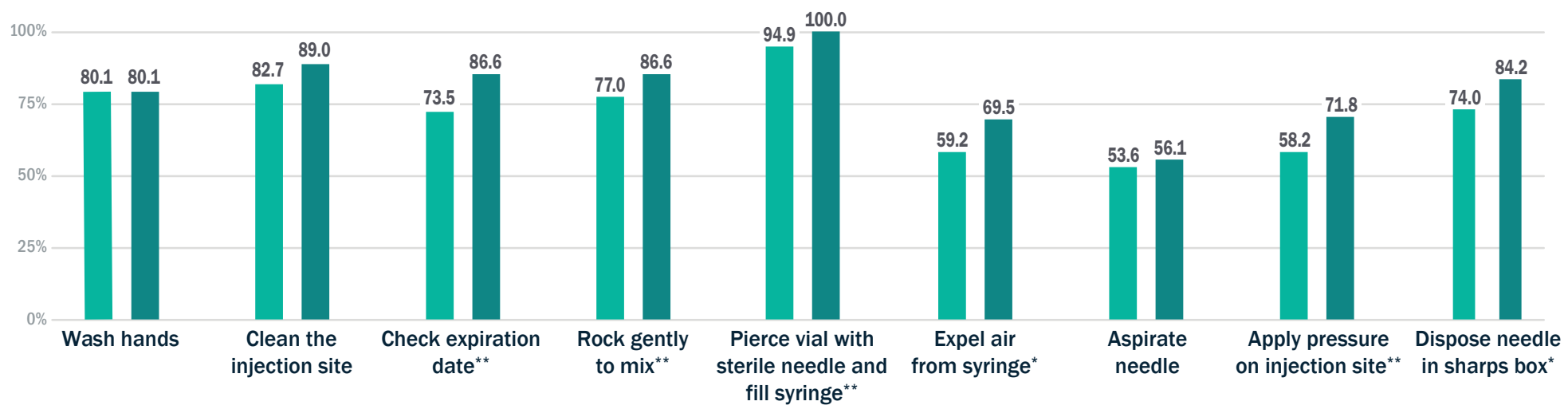

†Observations used for PPMVs in Bauchi, Cross River, Ebonyi and Kaduna

${ }^{*} p$-value $\leq 0.05 ;{ }^{* *} p$-value $\leq 0.01$ 1-month monitoring visit $(n=197) \quad$ 6-month monitoring visit $(n=164)$

Figure 6 shows the proportion of PPMVs who demonstrated the key DMPA-SC administration steps during the post-test and 1-month monitoring visit. As seen with DMPA-IM, most PPMVs (79\%) demonstrated the nine key steps for DMPA-SC administration at post-test. There was a decrease, however, in the proportion of PPMVs who demonstrated seven of the nine key DMPA-SC steps at the 1-month monitoring visit ( $\mathrm{n}=196)$. There were only two DMPA-SC steps, check expiration date $(91 \%$ to $100 \%$, p-value $\leq 0.01)$ and activate the Uniject ${ }^{\mathrm{TM}}$ ( $82 \%$ to $89 \%$, p-value $\leq 0.05$ ), where the proportion of PPMVs who named these steps increased from post-test to 1 -month monitoring visit.

A greater number of PPMVs demonstrated six of the nine key DMPA-SC steps at the 6-month monitoring visit compared to the 1-month monitoring visit (Figure 7). For example, when demonstrating the DMPA-SC steps, the proportion of PPMVs who inserted the Uniject straight into skin at a slight downward angle increased from 49 to 85 percent ( $\mathrm{p}$-value $\leq 0.01$ ). At the 6 -month monitoring visit, at least 75 percent of PPMVs demonstrated the key DMPA-SC steps.

\section{FIGURE 6. COMPARISON OF PPMV DEMONSTRATION OF KEY DMPA-SC ADMINISTRATION STEPS AT POST-TEST AND 1-MONTH MONITORING VISIT'}

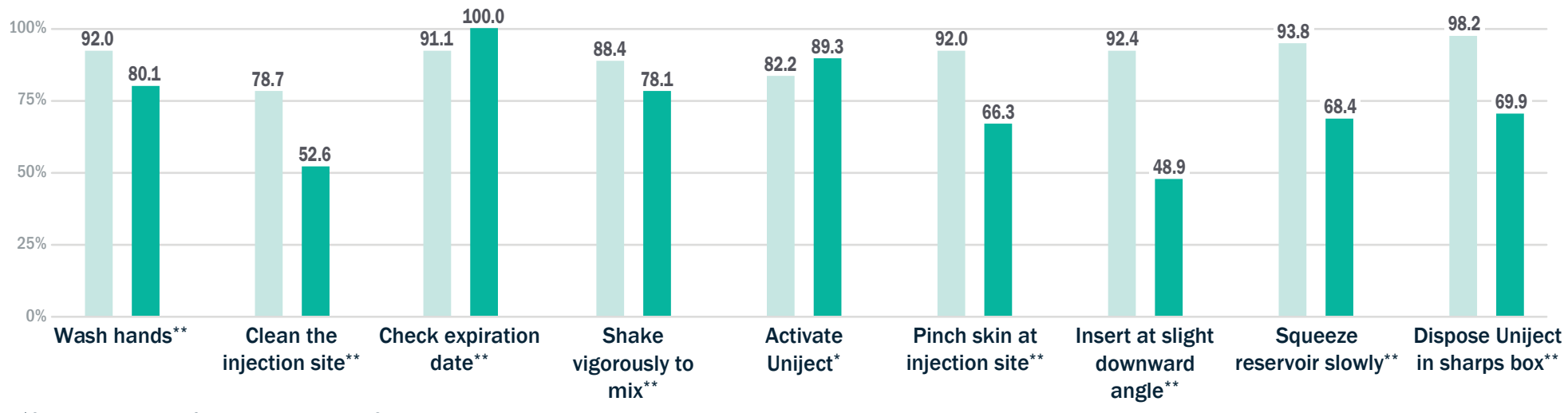

${ }^{\dagger}$ Observations used for PPMVs in Bauchi, Cross River, Ebonyi and Kaduna

${ }^{*} p$-value $\leq 0.05 ;{ }^{* *} p$-value $\leq 0.01 \square$ Post-test $(n=225) \quad$ 1-month monitoring visit $(n=196)$ 
FIGURE 7. COMPARISON OF PPMV DEMONSTRATION OF KEY DMPA-SC ADMINISTRATION STEPS AT 1-MONTH AND 6-MONTH MONITORING VISITS ${ }^{\dagger}$

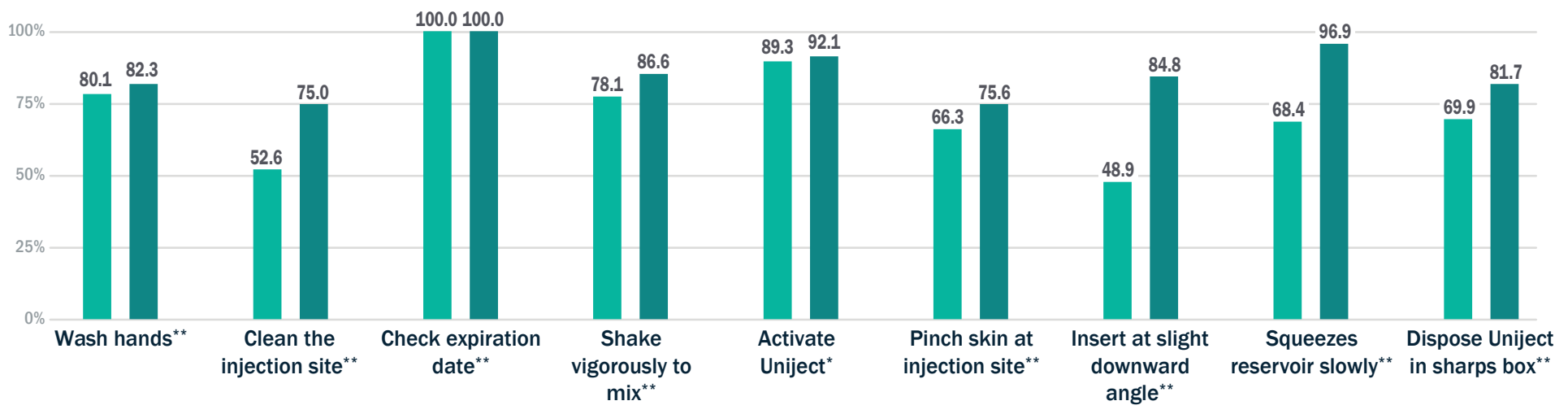

†Observations used for PPMVs in Bauchi, Cross River, Ebonyi and Kaduna

${ }^{*} p$-value $\leq 0.05 ;{ }^{* *} p$-value $\leq 0.01 \quad$ 1-month monitoring visit $(n=196) \quad$ 6-month monitoring visit $(n=164)$

\section{FIGURE 8. COMPARISON OF MONITORING TEAM OBSERVATIONS OF PPMVS' PRACTICES AT 1-MONTH AND 6-MONTH MONITORING VISITS ${ }^{\dagger}$}

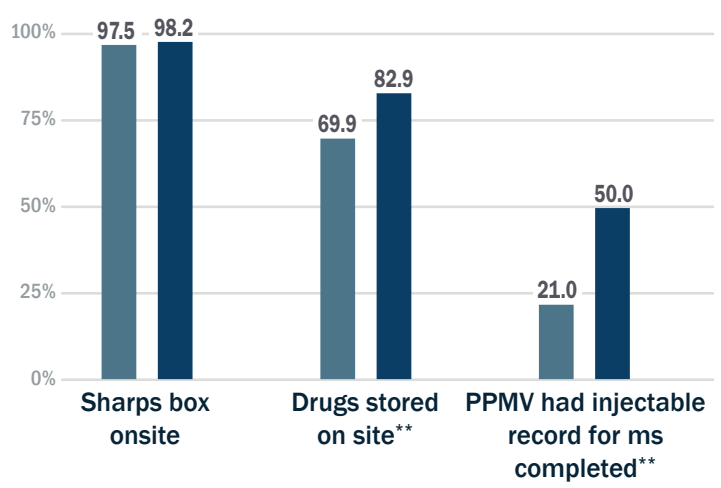

1-month monitoring visit ( $\mathrm{n}=196) \quad$ 6-month monitoring visit ( $\mathrm{n}=164)$ †Observations used for PPMVs in Bauchi, Cross River, Ebonyi and Kaduna "p-value $\leq 0.05 ;{ }^{* *}$-value $\leq 0.01$
Figure 8 presents the monitoring teams' observations of PPMVs and their shop during the 1- and 6-month monitoring visits. Almost all PPMVs in Bauchi, Cross River, Ebonyi and Kaduna were observed having a sharps disposal box in their shop at the 1-month and 6-month monitoring visits ( $98 \%$ each). There was an increase in the proportion of PPMVs who were observed properly storing the drugs in their shop from 70 percent at the 1 -month monitoring visit to 83 percent at the 6-month monitoring visit ( $\mathrm{p}$-value $\leq 0.01$ ), and those who kept injectable record form 21 percent at the 1 -month monitoring visit to 50 percent at the 6 -month monitoring visit ( $\mathrm{p}$-value $\leq 0.01$ ). One reason why only half of PPMVs kept injectable record forms may have been due to police and regulatory harassment, despite being authorized to provide injectable contraceptives under the study. During the monitoring visits, many PPMVs cited police harassment for why they did not keep up-to-date records. Regulatory and police representatives periodically visit patent medicine shops to ensure PPMVs are not selling unauthorized commodities, or for extortion. This may explain why some PPMVs reported that they did not keep records of their injectable contraceptive services.

Most PPMVs administered injectable contraceptives to at least one client monthly. Eighty-seven percent of PPMVs in all six states $(\mathrm{n}=318)$ reported they had administered an injectable contraceptive to at least one client in the 30 days preceding the 3-month follow-up survey and 92 percent reported they had administered an injectable contraceptive to at least one client in the 30 days preceding the 9 -month follow-up survey. At the 9-month survey, the average number of injections provided in the previous 30 days was 2.4 for DMPA-IM, 1.3 for NET-EN and less than 1 for DMPA-SC (data not shown). No adverse events were reported over the course of the study. 


\section{There was no difference in knowledge and skills between PPMVs with and without previous health facility experience}

PPMVs were asked a series of unprompted questions to ascertain their knowledge on injectable contraceptives. Specifically, PPMVs were asked about the knowledge needed for counseling (side effects), screening (exclusionary health conditions), and providing injectable contraceptives (when a woman can start the injectable, post-administration instructions for clients, and needle safety practices). The results in Table 3 show there were no differences between those who had and who had not previously worked in a health facility for most of the knowledge indicators. At the post-test survey, there were statistically significant differences in knowledge for only 8 of 41 indicators. For example, 76 percent of PPMVs who had worked in a health facility named dizziness as a side effect compared to 62 percent of PPMVs who had not worked in a health facility ( $\mathrm{p}$-value $\leq 0.05$ ). Interestingly, PPMVs who had not previously worked in a health facility demonstrated higher knowledge for two of those eight indicators. Forty percent of PPMVs who had not worked in a health facility named current or bistory of stroke and 35 percent named liver tumor as exclusionary health conditions compared to only 26 percent and 20 percent of PPMVs who had worked in a health facility ( $\mathrm{p}$-value $\leq 0.05$; $\mathrm{p}$-value $\leq 0.01$ ). There were no differences in knowledge of the nine method characteristic indicators (reinjection frequency, injection device, and administration location on the body) for DMPA-IM, NET-EN and DMPA-SC between the two groups (data not shown).

\section{TABLE 3. COMPARISON OF PPMV INJECTABLE KNOWLEDGE AMONG THOSE WHO HAD AND HAD NOT PREVIOUSLY WORKED IN A HEALTH FACILITY, POST-TEST SURVEY ${ }^{\dagger}$}

\begin{tabular}{|c|c|c|}
\hline & $\begin{array}{l}\text { PPMVs who had previously } \\
\text { worked in a facility }(n=95)\end{array}$ & $\begin{array}{l}\text { PPMVs who had not previously } \\
\text { worked in a facility }(n=203)\end{array}$ \\
\hline & \multicolumn{2}{|c|}{ Percentage } \\
\hline \multicolumn{3}{|l|}{ What are the common side effects of the injectable? } \\
\hline Change in menstruation & 96.8 & 91.6 \\
\hline Headache & 85.3 & 84.2 \\
\hline Weight gain & 64.2 & 55.7 \\
\hline Dizziness* ${ }^{*}$ & 75.8 & 62.1 \\
\hline Delayed return to fertility & 27.4 & 24.6 \\
\hline Temporary mild/moderate skin irritation ** & 40.0 & 18.7 \\
\hline Decrease in sex drive & 22.1 & 21.2 \\
\hline \multicolumn{3}{|c|}{ Under what health conditions should a woman NOT be provided the injectable? } \\
\hline High blood pressure $(160 / 100)^{\star}$ & 82.1 & 70.4 \\
\hline History of breast cancer & 65.3 & 70.0 \\
\hline Diabetes & 61.1 & 62.6 \\
\hline Current/history of stroke or ischaemic heart disease ${ }^{*}$ & 26.3 & 40.4 \\
\hline Multiple risk factors for cardiovascular disease & 40.0 & 37.0 \\
\hline Liver tumor ${ }^{* *}$ & 20.0 & 35.0 \\
\hline Unexplained vaginal bleeding & 38.9 & 32.0 \\
\hline Breastfeeding up to $4 / 6^{\ddagger}$ weeks postpartum & 25.3 & 18.7 \\
\hline Rheumatic disease & 15.8 & 19.7 \\
\hline Migraines with aura worsening with injectable use & 14.7 & 16.3 \\
\hline Deep vein thrombosis & 7.4 & 11.8 \\
\hline \multicolumn{3}{|l|}{ When can a woman start the injectable method? } \\
\hline When she is not pregnant & 67.4 & 56.7 \\
\hline $4 / 6^{\ddagger}$ weeks after childbirth & 66.3 & 63.1 \\
\hline Within $1^{\text {st }} 7$ days of a menstrual cycle w/out back-up method & 31.6 & 30.1 \\
\hline
\end{tabular}




\begin{tabular}{|c|c|c|}
\hline & $\begin{array}{l}\text { PPMVs who had previously } \\
\text { worked in a facility }(n=95)\end{array}$ & $\begin{array}{l}\text { PPMVs who had not previously } \\
\text { worked in a facility }(n=203)\end{array}$ \\
\hline & \multicolumn{2}{|c|}{ Percentage } \\
\hline After $1^{\text {st }} 7$ days of a menstrual cycle w/ back-up method & 27.4 & 37.0 \\
\hline Immediately after a miscarriage or abortion ${ }^{*}$ & 29.5 & 19.2 \\
\hline Immediately after stopping another method ${ }^{*}$ & 30.5 & 17.7 \\
\hline \multicolumn{3}{|c|}{ What instructions should be provided to women after administering the injection? } \\
\hline When to return for next injection & 90.5 & 96.1 \\
\hline Do not rub the injection site & 70.5 & 60.1 \\
\hline Provide information about possible side effects & 64.1 & 58.1 \\
\hline Go to health facility if concerns/questions about the method & 10.5 & 9.4 \\
\hline Go to health facility if any significant changes in health & 17.9 & 17.2 \\
\hline Go to health facility if suspicion about pregnancy & 11.6 & 7.9 \\
\hline \multicolumn{3}{|l|}{ What are the ways to handle needles/syringes safely? } \\
\hline Don't touch the needles & 75.8 & 72.9 \\
\hline Don't recap the needle & 71.6 & 68.0 \\
\hline Discard needle immediately after use ${ }^{\star *}$ & 80.0 & 61.6 \\
\hline Ensure sharps are disposed of in sharps box & 72.6 & 68.5 \\
\hline Don't overfill sharps box & 42.3 & 35.5 \\
\hline \multicolumn{3}{|c|}{ What precautions should be taken to prevent infection from a needle stick injury? } \\
\hline Wash hands with soap and water immediately & 75.8 & 69.0 \\
\hline Use sharps box for needles and sharps & 60.0 & 56.2 \\
\hline Handle needles carefully & 63.2 & 58.1 \\
\hline \multicolumn{3}{|l|}{ What should be done in the case of a needle stick injury? } \\
\hline Wash wound with soap and water & 85.3 & 86.2 \\
\hline Ensure nothing is put on wound site after cleaning it & 25.3 & 17.7 \\
\hline Apply plaster bandage to wound site & 11.6 & 5.4 \\
\hline
\end{tabular}

${ }^{*}$ p-value $\leq 0.05 ;{ }^{* *}$-value $\leq 0.01$

${ }^{\dagger}$ Comparisons used for PPMVs in all states except Oyo due to respondent ID error

₹ The WHO guidelines for injectable use and breastfeeding were updated during the study's implementation. PPMVs in the first two states were trained using the 6-week standard and PPMVs in the additional four states were trained using the 4-week standard.

Similar trends were observed at the 9-month follow-up survey (Table 4). Results shows that there were no significant differences in (a) knowledge of injectable side effects, (b) how to handle needles safely, (c) how to prevent infection from a needle stick injury, or (d) what should be done in case of a needlestick injury among those who had and had not previously worked in a health facility. Of the 41 knowledge indicators, PPMVs who had previously worked in a health facility demonstrated higher knowledge for only four of those indicators: (1) diabetes as an exclusionary health condition; (2) a woman can start the injectable within first seven days of her menstrual cycle without a back-up method; (3) when to return for the next injection; and, (4) provide information about possible side effects as post-administration information for clients.

\section{TABLE 4. COMPARISON OF PPMV INJECTABLE KNOWLEDGE AMONG THOSE WHO HAD AND HAD NOT PREVIOUSLY WORKED IN A HEALTH FACILITY, 9-MONTH FOLLOW-UP SURVEY ${ }^{\dagger}$}

\begin{tabular}{|c|c|c|}
\hline & $\begin{array}{l}\text { PPMVs who had previously } \\
\text { worked in a facility }(n=84)\end{array}$ & $\begin{array}{l}\text { PPMVs who had not previously } \\
\text { worked in a facility }(n=181)\end{array}$ \\
\hline & \multicolumn{2}{|c|}{ Percentage } \\
\hline \multicolumn{3}{|c|}{ What are the common side effects of the injectable? } \\
\hline Change in menstruation & 90.5 & 90.6 \\
\hline Headache & 85.7 & 81.2 \\
\hline Weight gain & 81.0 & 71.8 \\
\hline
\end{tabular}




\begin{tabular}{|c|c|c|}
\hline & $\begin{array}{l}\text { PPMVs who had previously } \\
\text { worked in a facility }(n=84)\end{array}$ & $\begin{array}{l}\text { PPMVs who had not previously } \\
\text { worked in a facility }(n=181)\end{array}$ \\
\hline & \multicolumn{2}{|c|}{ Percentage } \\
\hline Dizziness & 57.1 & 57.5 \\
\hline Delayed return to fertility & 17.9 & 17.7 \\
\hline Temporary mild/moderate skin irritation & 34.5 & 24.9 \\
\hline Decrease in sex drive & 23.8 & 20.4 \\
\hline \multicolumn{3}{|c|}{ Under what health conditions should a woman NOT be provided the injectable? } \\
\hline High blood pressure $(160 / 100)^{*}$ & 67.9 & 63.5 \\
\hline History of breast cancer & 52.4 & 57.5 \\
\hline Diabetes $^{\star *}$ & 82.1 & 63.0 \\
\hline Current/history of stroke or ischaemic heart disease & 33.3 & 27.6 \\
\hline Multiple risk factors for cardiovascular disease & 22.6 & 28.7 \\
\hline Liver tumor & 29.8 & 27.1 \\
\hline Unexplained vaginal bleeding & 31.0 & 27.1 \\
\hline Breastfeeding up to $4 / 6^{\ddagger}$ weeks postpartum & 29.8 & 24.9 \\
\hline Rheumatic disease & 4.8 & 8.9 \\
\hline Migraines with aura worsening with injectable use & 22.6 & 16.6 \\
\hline Deep vein thrombosis & 16.7 & 15.5 \\
\hline \multicolumn{3}{|l|}{ When can a woman start the injectable method? } \\
\hline When she is not pregnant & 82.1 & 72.4 \\
\hline $4 / 6^{\ddagger}$ weeks after childbirth & 66.7 & 58.0 \\
\hline Within $1^{\text {st }} 7$ days of a menstrual cycle w/out back-up method ${ }^{* *}$ & 54.8 & 37.6 \\
\hline After $1^{\text {st }} 7$ days of a menstrual cycle $w /$ back-up method & 26.2 & 37.0 \\
\hline Immediately after a miscarriage or abortion & 23.8 & 18.8 \\
\hline Immediately after stopping another method & 15.5 & 12.7 \\
\hline \multicolumn{3}{|c|}{ What instructions should be provided to women after administering the injection? } \\
\hline When to return for next injection ${ }^{*}$ & 96.4 & 88.4 \\
\hline Do not rub the injection site & 72.6 & 63.0 \\
\hline Provide information about possible side effects ${ }^{*}$ & 76.2 & 63.0 \\
\hline Go to health facility if concerns/questions about the method & 3.6 & 9.9 \\
\hline Go to health facility if any significant changes in health & 26.2 & 20.1 \\
\hline Go to health facility if suspicion about pregnancy & 13.1 & 6.6 \\
\hline \multicolumn{3}{|l|}{ What are the ways to handle needles/syringes safely? } \\
\hline Don't touch the needles & 81.0 & 78.5 \\
\hline Don't recap the needle & 81.0 & 79.0 \\
\hline Discard needle immediately after use & 54.8 & 58.6 \\
\hline Ensure sharps are disposed of in sharps box & 71.4 & 75.1 \\
\hline Don't overfill sharps box & 17.9 & 18.8 \\
\hline \multicolumn{3}{|c|}{ What precautions should be taken to prevent infection from a needle stick injury? } \\
\hline Wash hands with soap and water immediately & 81.0 & 68.0 \\
\hline Use sharps box for needles and sharps & 62.4 & 67.9 \\
\hline Handle needles carefully & 78.6 & 71.8 \\
\hline \multicolumn{3}{|l|}{ What should be done in the case of a needle stick injury? } \\
\hline Wash wound with soap and water & 82.1 & 85.6 \\
\hline Ensure nothing is put on wound site after cleaning it & 28.6 & 21.0 \\
\hline Apply plaster bandage to wound site & 15.5 & 15.5 \\
\hline
\end{tabular}

${ }^{*} p$-value $\leq 0.05 ;{ }^{* *} p$-value $\leq 0.01$

${ }^{\dagger}$ Comparisons used for PPMVs in all states except Oyo due to respondent ID error

‡ The WHO guidelines for injectable use and breastfeeding were updated during the study's implementation. PPMVs in the first two states were trained using the 6-week standard and PPMVs in the additional four states were trained using the 4-week standard. 
Figures 9, 10, 11 and 12 show the differences in PPMV demonstration of key DMPA-IM and DMPA-SC administration steps between those with and without health facility experience at the post-test and 6-month monitoring visits. As seen with the knowledge indicators, PPMVs without health facility experience demonstrated comparable skills for DMPA-IM and DMPA-SC administration to those with health facility experience at both time points. For example, only for two DMPA-IM steps, withdraw contents and expel air from the syringe and aspirate needle, and one key DMPA-SC step, check expiration date, did a significantly higher proportion of PPMVs with health facility experience demonstrate these steps compared to those without health facility experience at the post-test observation (Figures 9 and 10). At the 6-month monitoring visit, however, there were no differences for eight of the nine key DMPA-IM steps and seven of the nine DMPA-SC steps. For the steps where there were significant differences, a higher proportion of PPMVs without health facility experience demonstrated these steps (dispose of needle for DMPA-IM and guide client to select injection site and activate Uniject for DMPA-SC) compared to those with health facility experience (Figures 11 and 12).

\section{FIGURE 9. COMPARISON OF PPMV DEMONSTRATION OF KEY DMPA-IM ADMINISTRATION STEPS AMONG THOSE WHO HAD AND HAD NOT PREVIOUSLY WORKED IN A HEALTH FACILITY, POST-TEST}

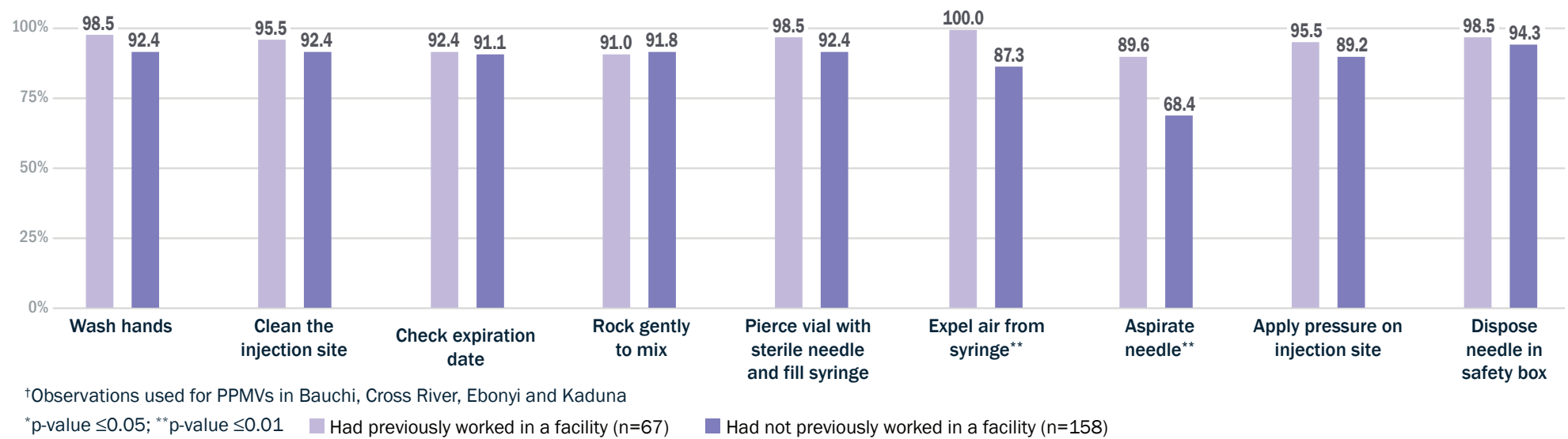

\section{FIGURE 10. COMPARISON OF PPMV DEMONSTRATION OF KEY DMPA-SC ADMINISTRATION STEPS AMONG THOSE WHO HAD AND HAD NOT PREVIOUSLY WORKED IN A HEALTH FACILITY, POST-TEST}

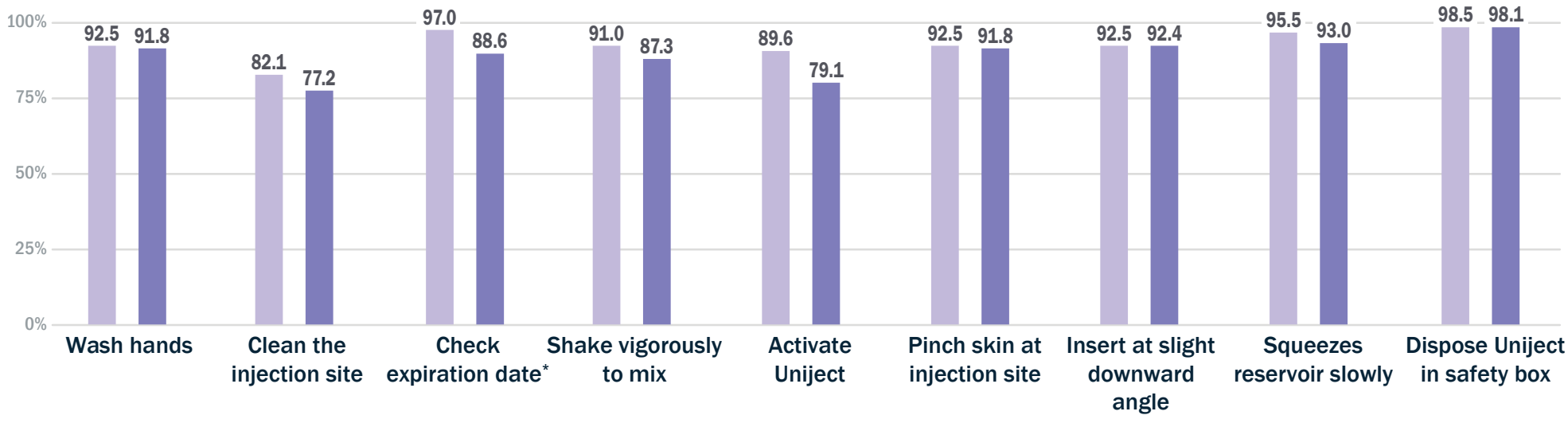

+Observations used for PPMVs in Bauchi, Cross River, Ebonyi and Kaduna ${ }^{*}$-value $\leq 0.05 ;{ }^{* *} p$-value $\leq 0.01 \quad$ Had previously worked in a facility $(n=67)$ 
FIGURE 11. COMPARISON OF PPMV DEMONSTRATION OF KEY DMPA-IM ADMINISTRATION STEPS AMONG THOSE WHO HAD AND HAD NOT PREVIOUSLY WORKED IN A HEALTH FACILITY, 6-MONTH MONITORING VISIT

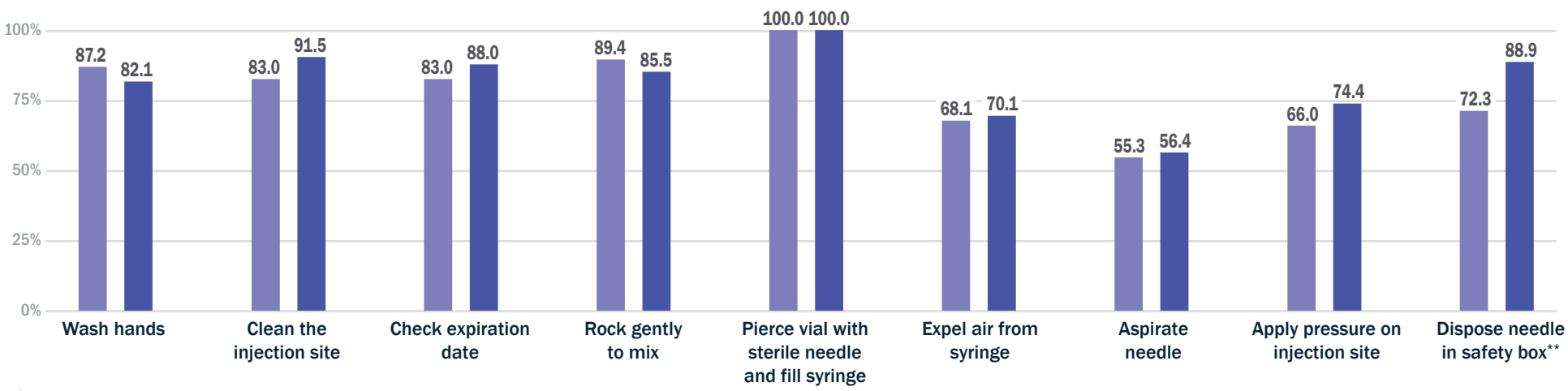

†Observations used for PPMVs in Bauchi, Cross River, Ebonyi and Kaduna

*p-value $\leq 0.05 ;{ }^{* *} p$-value $\leq 0.01 \quad$ Had previously worked in a facility $(n=47)$

Had not previously worked in a facility $(n=117)$

FIGURE 12. COMPARISON OF PPMV DEMONSTRATION OF KEY DMPA-SC ADMINISTRATION STEPS AMONG THOSE WHO HAD AND HAD NOT PREVIOUSLY WORKED IN A HEALTH FACILITY, 6-MONTH MONITORING VISIT

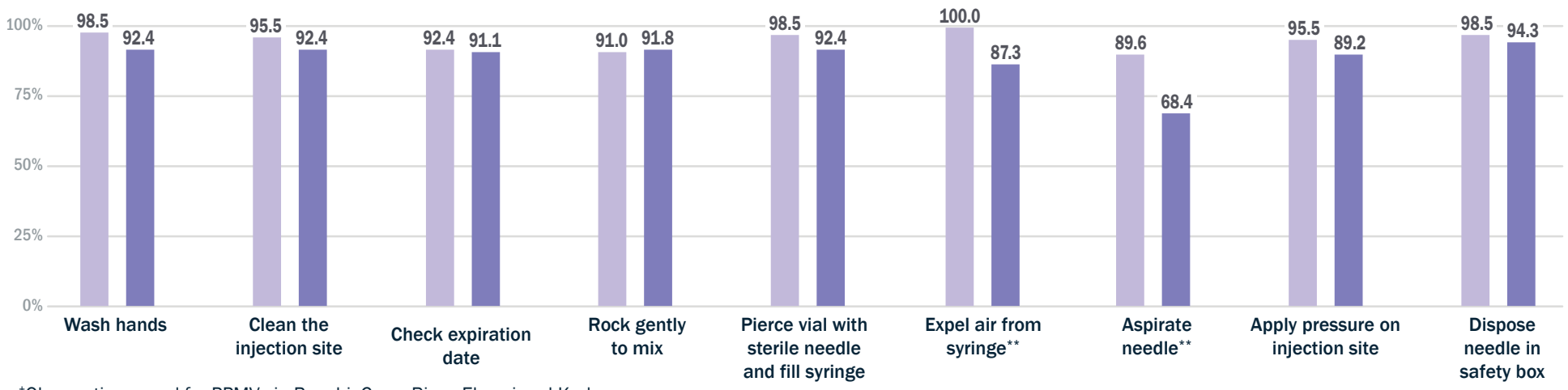

${ }^{\dagger}$ Observations used for PPMVs in Bauchi, Cross River, Ebonyi and Kaduna

"p-value $\leq 0.05 ;{ }^{* *}$-value $\leq 0.01$ Had previously worked in a facility $(n=67) \quad$ Had not previously worked in a facility ( $\left.n=158\right)$

\section{PPMVs knowledge of injectable contraceptive characteristics was higher after the training and was retained nine months after the training}

Knowledge increased from the pretest to post-test survey

PPMVs were asked a series of unprompted questions to ascertain their knowledge on injectable contraceptives during the pre-, post- and 9-month surveys. Significant increases in PPMVs' knowledge related to counseling, screening and administration of the injectable contraceptive method were observed on 47 of 50 knowledge indicators from the pretest to post-test survey. Knowledge doubled for 32 indicators and increased by at least half for eight indicators. Despite these increases, knowledge remained below 50 percent for 21 indicators at the post-test survey.

Figure 13 shows PPMV knowledge of seven common side effects of the injectable method at the pretest and post-test surveys. At the pretest survey, less than one-third of PPMVs spontaneously named headache (23\%), weight gain (32\%), dizziness (19\%), delayed return to fertility (8\%), temporary skin irritation (1\%) or decrease in sex drive 
$(2 \%)$ as common side effects of the injectable method. At the post-test survey, knowledge on all seven common side effects increased significantly. Larger increases were seen for: (1) change in menstruation from 64 percent to 93 percent; (2) headache from 23 percent to 79 percent; (3) weight gain from 32 percent to 60 percent; and (4) dizziness from 19 percent to 56 percent. For the remaining three side effects, the percentage point increases in knowledge at post-test were not as large and knowledge remained low at approximately $20 \%$.

\section{FIGURE 13. KNOWLEDGE COMPARISONS OF SEVEN COMMON SIDE EFFECTS OF THE INJECTABLE AT PRETEST AND POST-TEST SURVEYS ${ }^{\dagger}$}

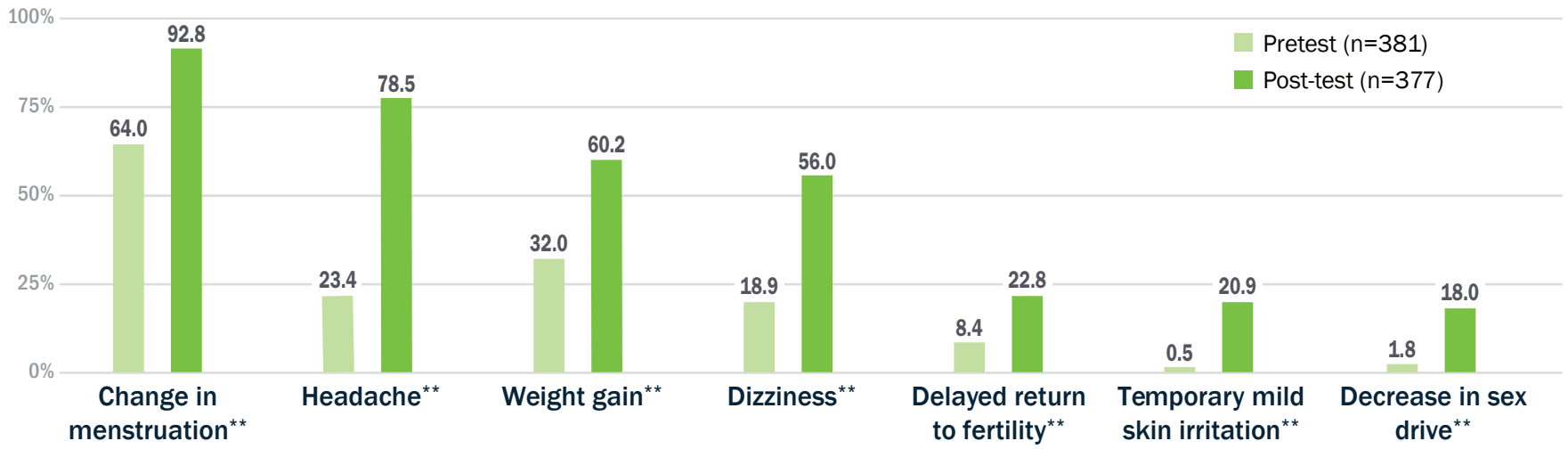

${ }^{\dagger}$ Post-training surveys asked 1 month after training in the $1^{\text {st }}$ two states and immediately after the training in the additional four states.

"p-value $\leq 0.05 ;{ }^{* *} \mathrm{p}$-value $\leq 0.01$

Figure 14 shows that pretest and post-test survey results of 11 exclusionary health conditions of the injectable contraceptive. At the pretest survey, 41 percent of PPMVs named bigh blood pressure as an exclusionary health condition. Fewer spontaneously named any of the other health conditions ${ }^{8}$, ranging from 19 percent saying diabetes to 3 percent migraines with aura or deep vein thrombosis. At the post-test survey, knowledge on all 11 exclusionary health conditions increased significantly, yet eight health conditions were mentioned by fewer than 50 percent.

\section{FIGURE 14. KNOWLEDGE COMPARISONS OF 11 EXCLUSIONARY HEALTH CONDITIONS OF THE INJECTABLE AT PRETEST AND POST-TEST SURVEYS ${ }^{\dagger}$}

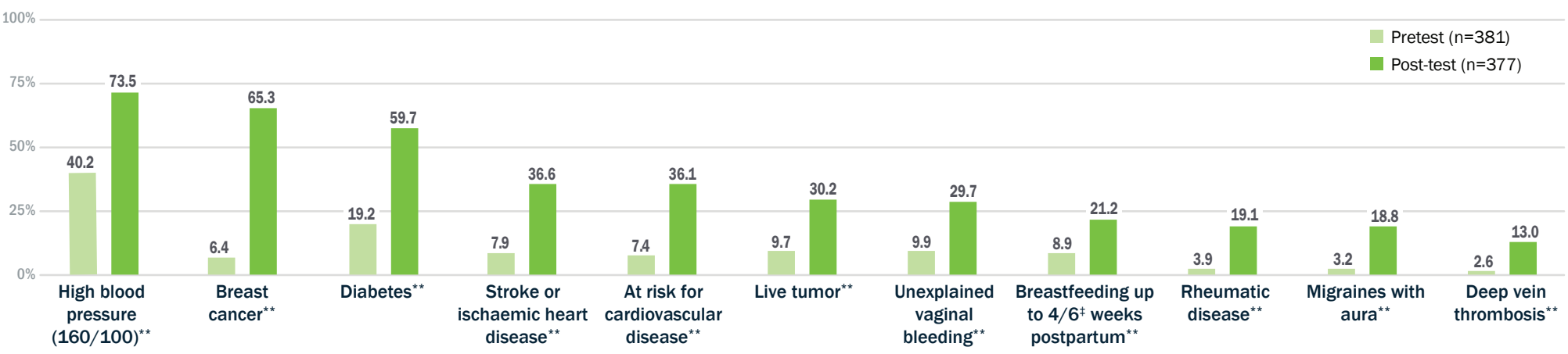

†Post-training surveys asked 1 month after training in the $1^{\text {st }}$ two states and immediately after the training in the additional four states.

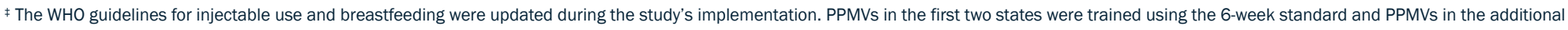
four states were trained using the 4-week standard.

*p-value $\leq 0.05 ;{ }^{* *}$-value $\leq 0.01$

8 For the purposes of the study, PPMVs were authorized to administer clients were in category one based on the WHO Medical Eligibility Criteria. 
Figure 15 presents PPMVs' knowledge of when a woman can begin the injectable. At the pretest survey, only 33 percent of PPMVs said if she is not pregnant and 19 percent said within the first seven days of her menstrual cycle without a back-up method for when a woman can start the injectable. At the post-test survey, these two indicators increased to 58 percent and 41 percent, respectively. Statistically significant increases in knowledge were observed for all knowledge indicators in Figure 15 except after first seven days of a menstrual cycle with back-up method. After the training, few PPMVs spontaneously recalled that the injection could be used immediately after a miscarriage or abortion (18\%) or immediately after stopping another method (18\%).

\section{FIGURE 15. KNOWLEDGE COMPARISONS OF WHEN A WOMAN CAN START THE INJECTABLE AT PRETEST AND POST-TEST SURVEYS ${ }^{\dagger}$}

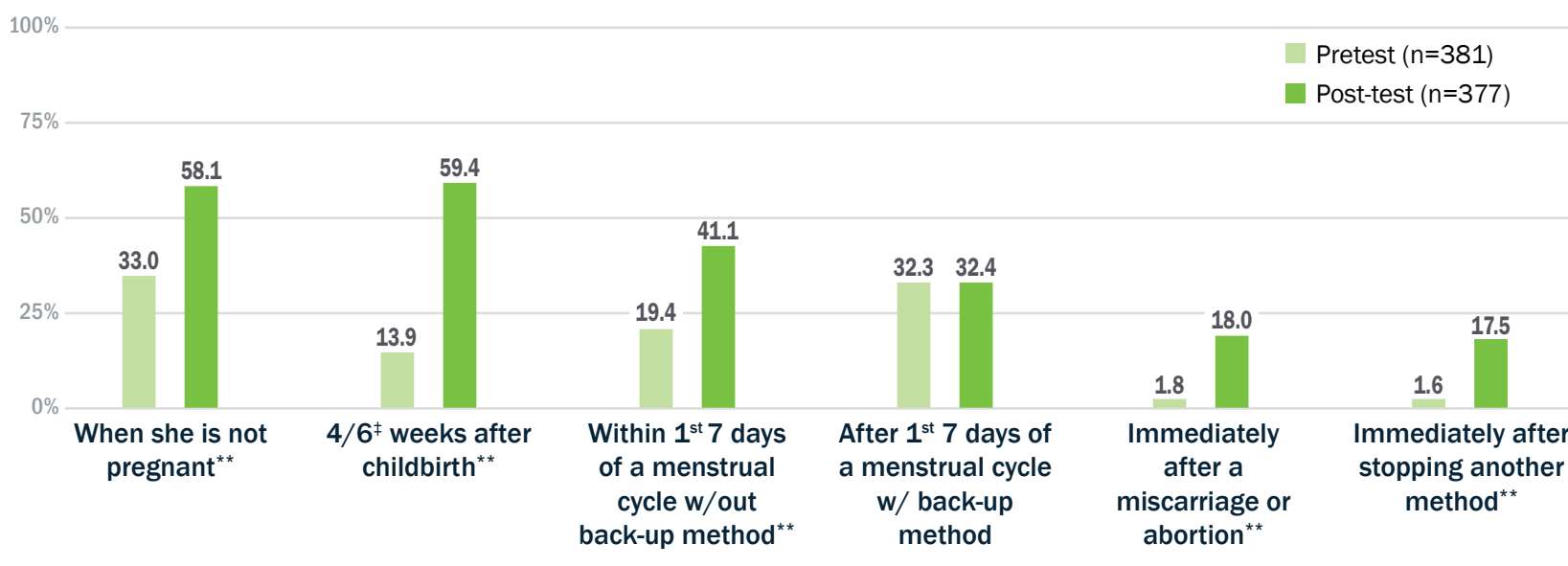

${ }^{\dagger}$ Post-training surveys asked 1 month after training in the $1^{\text {st }}$ two states and immediately after the training in the additional four states. ‡The WHO guidelines for injectable use and breastfeeding were updated during the study's implementation. PPMVs in the first two states were trained using the 6-week standard and PPMVs in the additional four states were trained using the 4-week standard. ${ }^{*}$ p-value $\leq 0.05 ;{ }^{* *}$ p-value $\leq 0.01$

Figure 16 shows PPMV responses to the information that should be offered to clients after receiving an injectable. Across the six indicators at the pretest survey, the proportion of PPMVs who knew this information ranged from 40 percent for when to return for the next injection to 5 percent for going to bealth facility for significant changes in health. At the post-test survey, nearly all (92\%) of PPMVs knew to tell their client when to return for their next injection. Roughly two-thirds (66\%) of PPMVs knew to tell their client not to rub the injection site and more than half $(60 \%)$ knew to provide information about possible side effects. Fewer PPMVs, however, said that clients should go to the health facility if significant changes in health (15\%), go to health facility if concerns/ questions about methods (9\%), or to go to the health facility if suspicion of pregnancy (7\%). When comparing these results to client responses in Figure 26, 91 percent of clients reported the PPMV told them what to do if they had a problem with the injectable at their first injection ( $n=561)$. Eighty-seven percent of clients reported they were instructed to return to the PPMV if they experienced any problems (data not shown).

Despite knowledge gains for 29 of the 30 indicators listed in Figures 13-16, knowledge remained low for some indicators. However, most PPMVs (86\%) in Bauchi, Cross River, Ebonyi, and Kaduna reported using job aids with their clients, suggesting PPMVs may refer to job aids to assist in remembering long lists of information (e.g. side effects and exclusionary health conditions) when providing injectable contraceptive services.

PPMVs were also asked about the characteristics of different injectable methods as shown in Figure 17. Results show that key knowledge of DMPA-IM, DMPA-SC, and NET-EN ${ }^{9}$ was significantly higher at the post-test

9 Only PPMVs in the additional four states were asked questions about NET-EN. 
than at the pretest survey. The greatest increases in knowledge were observed for DMPA-SC because a small proportion of PPMVs at pretest (5\%) knew about this method (data not shown). At the post-test survey, over 89 percent of PPMVs correctly named the (a) reinjection frequency, (b) at least one location on the body where the injectable can be administered, and (c) the device used to administer each of the three injectable methods.

FIGURE 16. KNOWLEDGE COMPARISONS OF POST-ADMINISTRATION INSTRUCTIONS AT PRETEST AND POST-TEST SURVEYS ${ }^{\dagger}$

When to return for next injection **

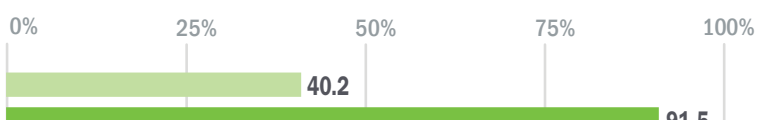

Do not rub the injection site ${ }^{*}$

Provide information about possible side effects ${ }^{\star \star}$

Go to health facility if any significant changes in health**

Go to health facility if concerns/questions about the method

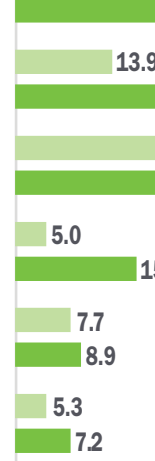

13.9

22.8 15.7

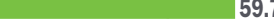

59.7

66.1

Pretest $(\mathrm{n}=381)$

Post-test $(n=377)$

${ }^{\dagger}$ Post-training survey asked 1 month after training in the $1^{\text {st }}$ two states and immediately after the training in the additional four states. "p-value $\leq 0.05 ;{ }^{* *} p$-value $\leq 0.01$

\section{FIGURE 17. KNOWLEDGE COMPARISONS OF KEY INJECTABLE METHOD CHARACTERISTICS AT PRETEST AND POST-TEST SURVEYS ${ }^{\dagger}$}

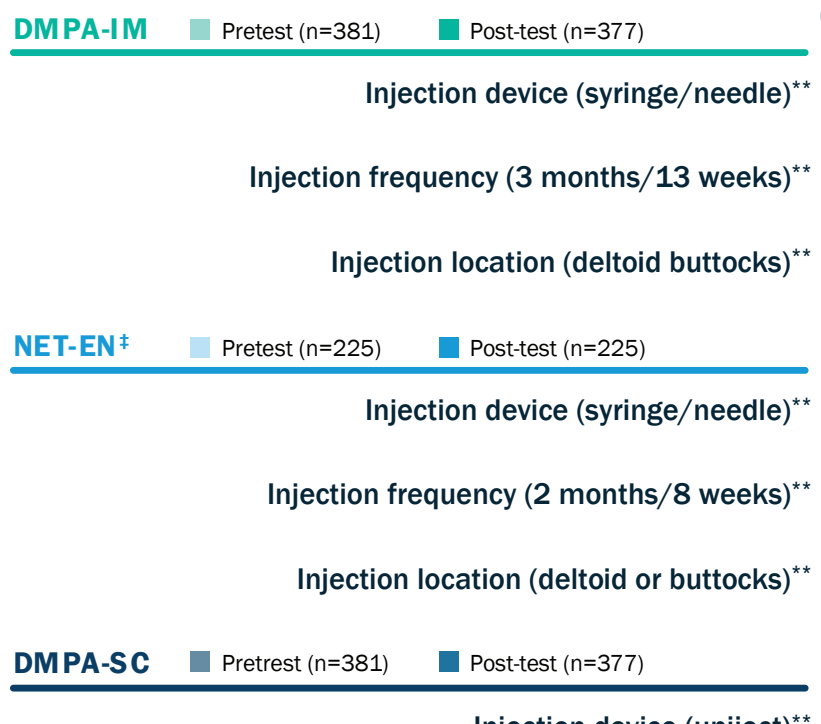

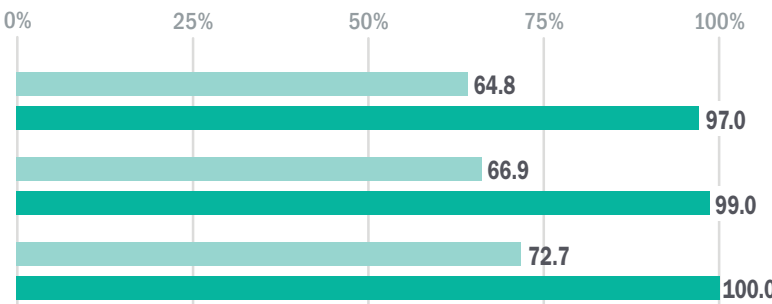

$$
\begin{aligned}
& \text { Injection device (uniject) } \\
& \text { Injection frequency (3 months/13 weeks) } \\
& \text { Injection location (back of arm, abdomen or front of thigh) }
\end{aligned}
$$
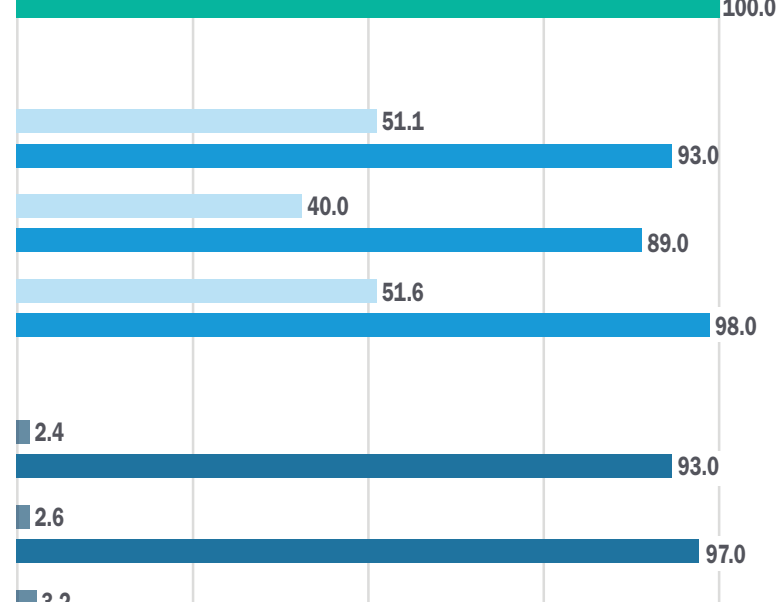

3.2

†Post-training surveys asked 1 month after training in the $1^{\text {st }}$ two states and immediately after the training in the additional four states. ${ }^{\ddagger}$ Asked of PPMVs in Bauchi, Cross River, Ebonyi and Kaduna only

"p-value $\leq 0.05$; ${ }^{* *} p$-value $\leq 0.01$ 
To understand PPMVs knowledge of needle safety practices, PPMVs were asked: (1) how to handle needles safely; (2) how to prevent infection from a needle stick injury; and (3) what to do in the event of a needle stick injury. Table 5 presents these results. For most of the indicators, fewer than 50 percent of PPMVs displayed knowledge before the training, but the percentage increased significantly after the training. For example, 72 percent of PPMVs at the post-test survey identified don't recap needles as one way to handle needles safely compared to 36 percent at the pretest survey ( $\mathrm{p}$-value $\leq 0.01$ ). Seventy-four percent at the post-test survey identified wash hands before and after administration to prevent infection compared to 31 percent at the pretest survey ( $\mathrm{p}$-value $\leq 0.01)$. The percentage of PPMVs with knowledge remained low at the post-test survey for ensure nothing is in the wound site $(18 \%)$ and decreased from 24 percent to 8 percent for apply plaster bandage to the wound site.

TABLE 5. KNOWLEDGE COMPARISONS OF NEEDLE SAFETY PRACTICES AT PRETEST AND POST-TEST SURVEYS ${ }^{\dagger}$

\begin{tabular}{|c|c|c|}
\hline & Pre-test $(n=381)$ & Post-test $(n=377)$ \\
\hline & \multicolumn{2}{|c|}{ Percentages } \\
\hline \multicolumn{3}{|l|}{ What are the ways to handle needles safely } \\
\hline Don't touch the needle ${ }^{* *}$ & 48.6 & 74.0 \\
\hline Don't recap the needle ${ }^{* *}$ & 29.7 & 65.8 \\
\hline Discard needle immediately after use ${ }^{\star *}$ & 35.7 & 71.9 \\
\hline Ensure sharps are disposed of in sharps box ${ }^{* *}$ & 32.6 & 69.8 \\
\hline Don't overfill sharps box ${ }^{* *}$ & 9.2 & 36.1 \\
\hline \multicolumn{3}{|c|}{ What precautions should be taken to prevent infection from needle stick injuries } \\
\hline $\begin{array}{l}\text { Wash hands with soap and water immediately } \\
\text { before and after administering the injectable }\end{array}$ & 30.5 & 73.5 \\
\hline Use sharps box for needles and sharps ${ }^{* *}$ & 35.7 & 59.7 \\
\hline Handle needles carefully* & 50.9 & 62.3 \\
\hline \multicolumn{3}{|l|}{ What should be done in the case of a needles stick injury } \\
\hline Wash wound with soap and water ${ }^{* *}$ & 36.8 & 82.5 \\
\hline Ensure nothing is put on wound site after cleaning it & 22.6 & 17.8 \\
\hline Apply plaster bandage to wound site ${ }^{* *}$ & 23.6 & 7.9 \\
\hline
\end{tabular}

*p-value $\leq 0.05 ;{ }^{* *}$ p-value $\leq 0.01$

†Post-training survey asked 1 month after training in the $1^{\text {st }}$ two states and immediately after the training in the additional four states.

\section{Knowledge retention from post-test to 9-month follow-up survey}

PPMVs retained knowledge of injectable contraceptive characteristics nine months after the training. Among the 50 indicators used to measure knowledge, from the post-test survey to the 9-month follow-up survey, knowledge increased for 13 of 50 indicators and was retained for 29 of 50 indicators. For 21 indicators, less than 50 percent of PPMVs displayed knowledge at the post-test survey.

Figures 18, 19, 20 and 21 show knowledge comparisons between the post-test and 9-month surveys for common side effects, exclusionary health conditions, when a woman can begin the injectable and post-administration instructions. Results show that there were no significant differences for most of the indicators between the post-test and 9-month follow-up surveys. In some cases, knowledge increased. For example, in Figure 18 knowledge of weight gain as a side effect increased from 60 percent at the post-test to 74 percent at the 9-month survey ( $\mathrm{p}$-value $\leq 0.01$ ). In Figure 20, PPMVs who said a woman can start the injectable method when she is not 
pregnant increased from 58 percent to 73 percent, and within the first seven days of her menstrual cycle without a back-up method increased from 41 percent to 55 percent ( $\mathrm{p}$-value $\leq 0.05$ ).

There were few decreases in knowledge between the post-test and 9-month surveys. In Figure 18, fewer PPMV identified delayed return to fertility as a side effect at the 9-month survey (15\%) compared to the post-test survey (23 percent, p-value $\leq 0.01)$. In Figure 19, fewer PPMVs named the following exclusionary health conditions at the 9-month survey compared to the post-test: history of breast cancer (65\% vs. $44 \%$ ), liver tumor (30\% vs. $23 \%$ ), and rheumatic disease (19\% vs. $6 \%$ ).

Figure 22 shows that PPMVs' knowledge of method-specific characteristics was the same between the posttest and 9-month surveys for six of the nine indicators. PPMVs' knowledge of the correct injection device used for DMPA-IM (syringe and needle) and for DMPA-SC (Uniject) increased from the post-test survey to 9-month survey. For example, 100 percent of PPMVs correctly named Uniject as the injection device for DMPA-SC compared to 93 percent at the post-test survey. Only for the DMPA-SC reinjection frequency (13 weeks) was there a decrease in knowledge between the 9-month and post-test survey. Still, 92 percent of PPMVs correctly named 13 weeks as the DMPA-SC re-injection frequency at the 9-month survey.

\section{FIGURE 18. KNOWLEDGE COMPARISONS OF SEVEN COMMON SIDE EFFECTS OF THE INJECTABLE AT POST-TEST ${ }^{\dagger}$ AND 9-MONTH SURVEYS}

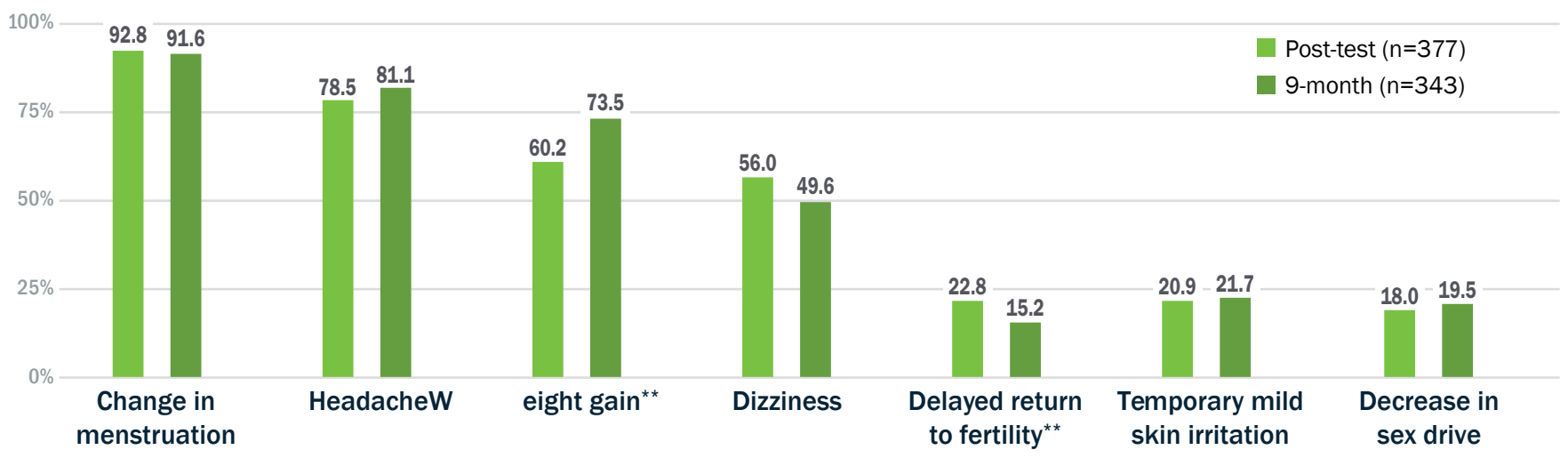

${ }^{\dagger}$ Post-training surveys asked 1 month after training in the $1^{\text {st }}$ two states and immediately after the training in the additional four states. *p-value $\leq 0.05 ;{ }^{* *} p$-value $\leq 0.01$

\section{FIGURE 19. KNOWLEDGE COMPARISONS OF 11 EXCLUSIONARY HEALTH CONDITIONS OF THE INJECTABLE AT POST-TEST ${ }^{\dagger}$ AND 9-MONTH SURVEYS}

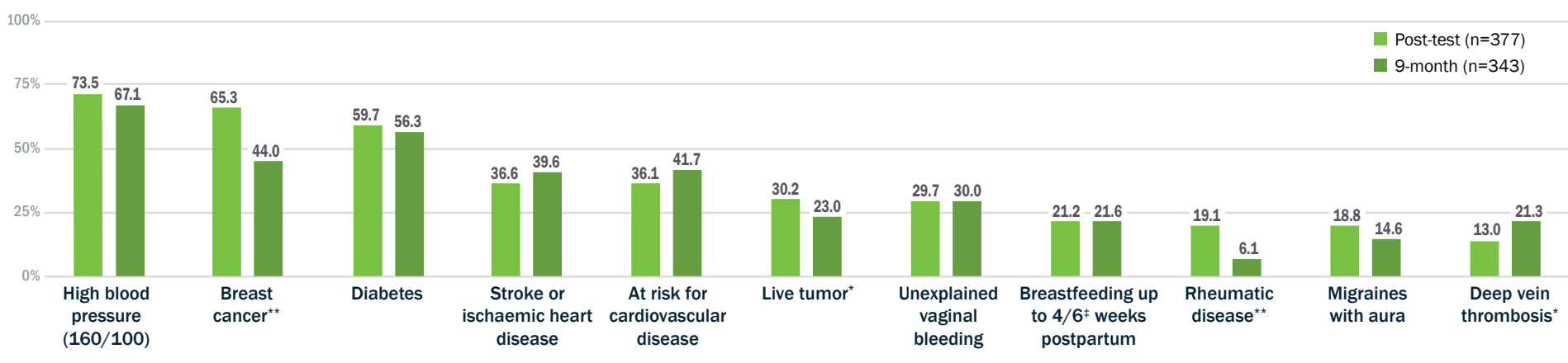




\section{FIGURE 20. KNOWLEDGE COMPARISONS OF WHEN A WOMAN CAN START THE INJECTABLE AT THE POST-TEST' ${ }^{\dagger}$ AND 9-MONTH SURVEYS}

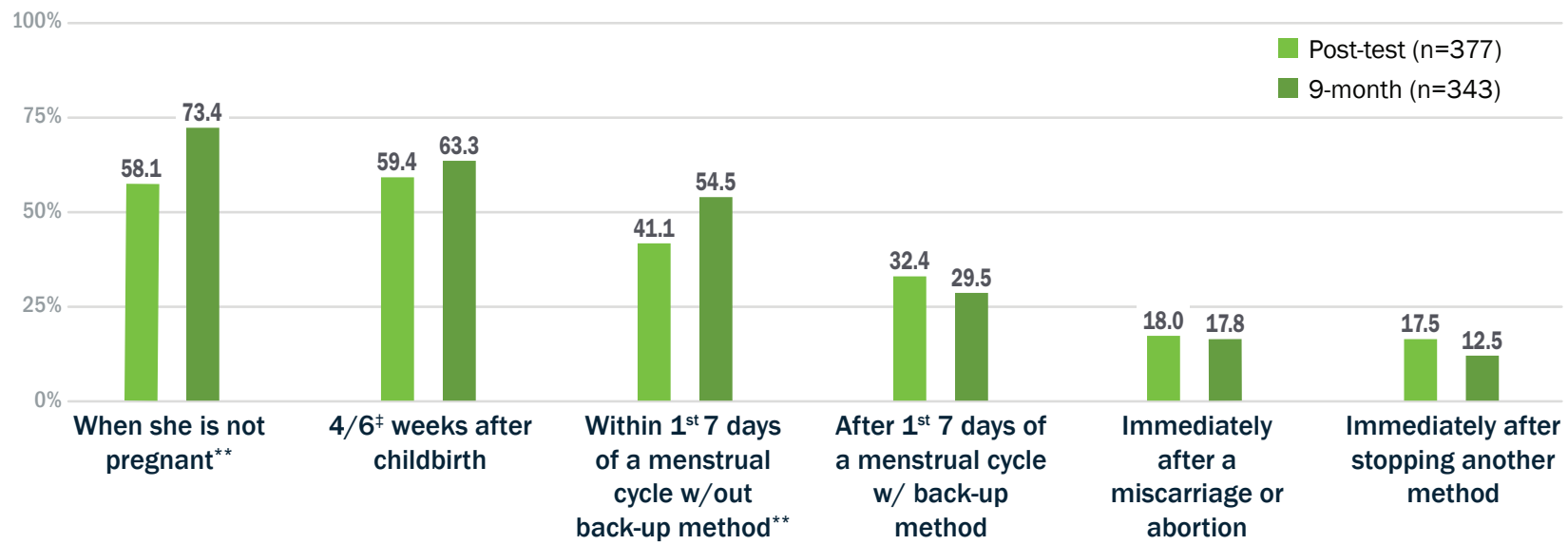

${ }^{\dagger}$ Post-training surveyss asked 1 month after training in the $1^{\text {st }}$ two states and immediately after the training in the additional four states. †The WHO guidelines for injectable use and breastfeeding were updated during the study's implementation. PPMVs in the first two states were trained using the 6-week standard and PPMVs in the additional four states were trained using the 4-week standard.

"p-value $\leq 0.05 ;{ }^{* *} p$-value $\leq 0.01$

\section{FIGURE 21. KNOWLEDGE COMPARISONS OF POST-ADMINISTRATION INSTRUCTIONS AT THE POST-} TEST $^{\dagger}$ AND 9-MONTH SURVEYS

When to return for next injection

Do not rub the injection site

Provide information about possible side effects

Go to health facility if concerns/questions about the method

Go to health facility if any significant changes in health**

Go to health facility if suspicion about pregnancy
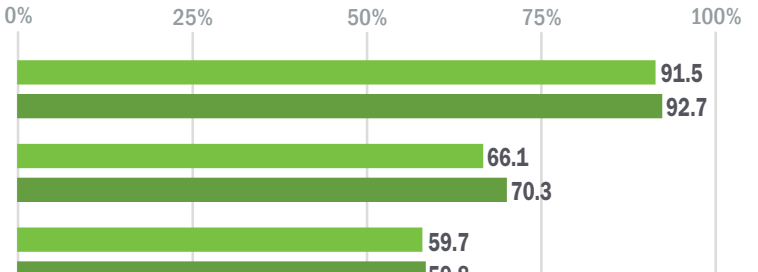

\section{7}

70.3
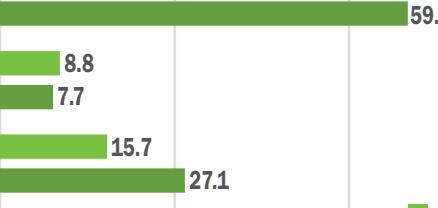

27.1

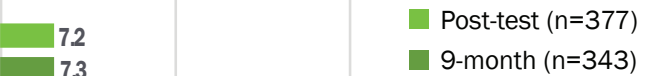

9-month $(n=343)$

${ }^{\dagger}$ Post-training survey asked 1 month after training in the $1^{\text {st }}$ two states and immediately after the training in the additional four states. "p-value $\leq 0.05 ;{ }^{* *}$-value $\leq 0.01$

Table 6 presents changes in PPMVs' knowledge of needle safety measures from the post-test and 9-month surveys. PPMVs' knowledge increased for many of the needle safety measures: a higher proportion of PPMVs named wash hands with soap and water $(72 \%$ compared to $60 \%$, p-value $\leq 0.01)$ and handle needles carefully $(80 \%$ compared to $62 \%$, p-value $\leq 0.01$ ) as ways to prevent infection from needle stick injury at the 9-month survey. Increases were also seen for three of five indicators to handle needles safely. Only for two indicators, discard needle immediately after use and don't overfill the sharps box, did the percentage of PPMVs with knowledge decrease from the post-test to 9-month survey (20 and 21 percentage point decrease, respectively). 
FIGURE 22. KNOWLEDGE COMPARISONS OF KEY INJECTABLE METHOD CHARACTERISTICS AT POSTTEST ${ }^{\dagger}$ AND 9-MONTH SURVEYS

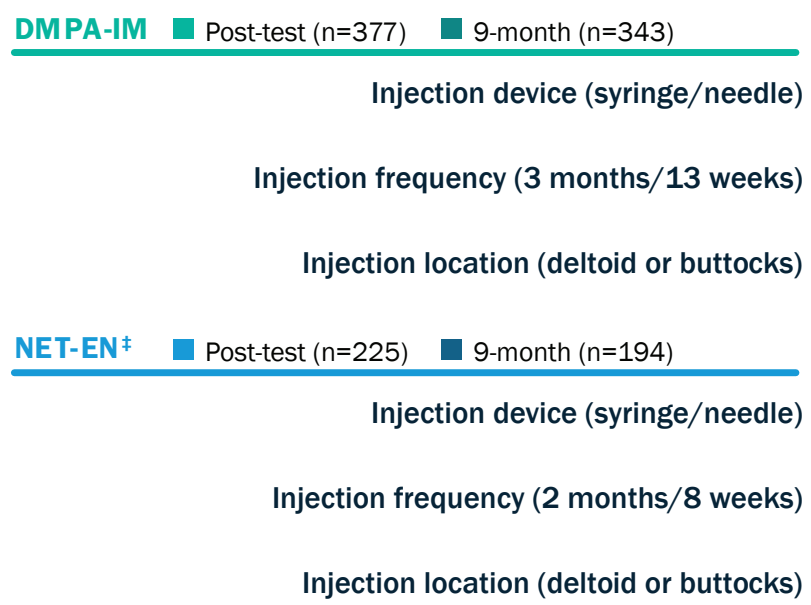

Injection location (back of arm, abdomen or front of thigh)
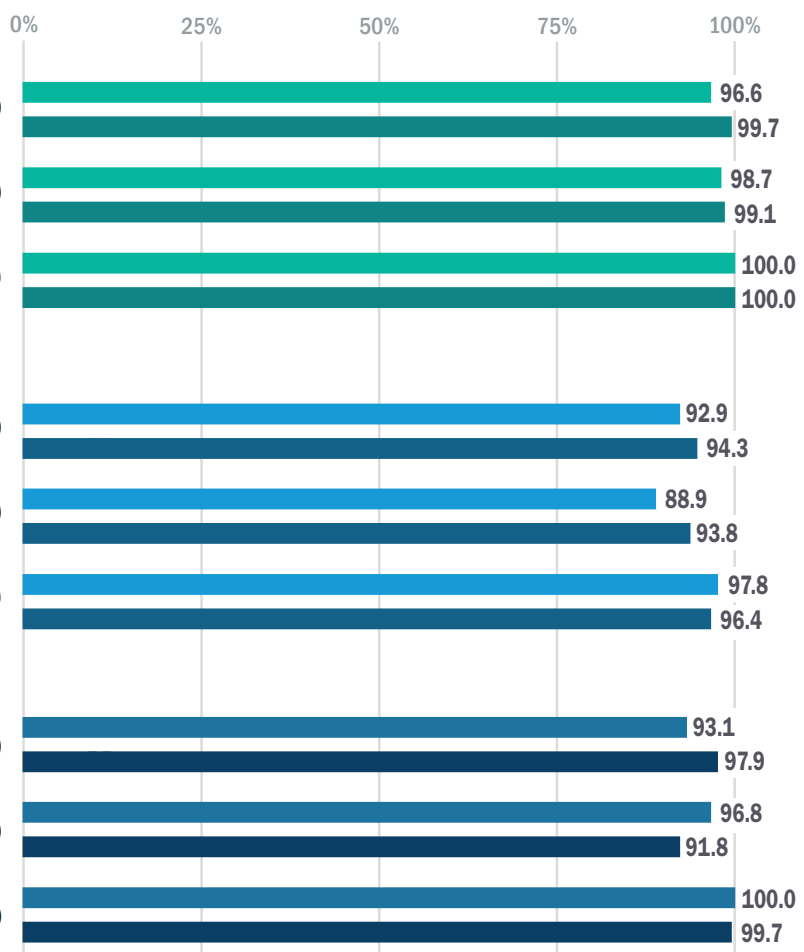

†Post-training surveys asked 1 month after training in the $1^{\text {st }}$ two states and immediately after the training in the additional four states. ${ }^{\ddagger}$ Asked of PPMVs in Bauchi, Cross River, Ebonyi and Kaduna only *p-value $\leq 0.05$; * p-value $\leq 0.01$

\section{TABLE 6. KNOWLEDGE COMPARISONS OF NEEDLE SAFETY PRACTICES AT POST-TEST ${ }^{\dagger}$ AND 9-MONTH SURVEYS}

\begin{tabular}{|c|c|c|}
\hline & Post-test $(n=377)$ & 9-month $(n=343)$ \\
\hline & \multicolumn{2}{|c|}{ Percentages } \\
\hline \multicolumn{3}{|l|}{ What are the ways to handle needles safely } \\
\hline Don't touch the needle ${ }^{* *}$ & 74.0 & 83.9 \\
\hline Don't recap the needle $e^{* *}$ & 65.8 & 84.3 \\
\hline Discard needle immediately after use ${ }^{\star *}$ & 71.9 & 51.9 \\
\hline Ensure sharps are disposed of in sharps box ${ }^{* *}$ & 69.8 & 79.9 \\
\hline Don't overfill sharps box ${ }^{* *}$ & 36.1 & 14.9 \\
\hline \multicolumn{3}{|c|}{ What precautions should be taken to prevent infection from needle stick injuries } \\
\hline $\begin{array}{l}\text { Wash hands with soap and water immediately before } \\
\text { and after administering the injectable }\end{array}$ & 73.5 & 78.4 \\
\hline Use sharps box for needles and sharps ${ }^{* *}$ & 59.7 & 72.3 \\
\hline Handle needles carefully ${ }^{\star *}$ & 62.3 & 79.9 \\
\hline \multicolumn{3}{|l|}{ What should be done in the case of a needle stick injury } \\
\hline Wash wound with soap and water ${ }^{*}$ & 82.5 & 88.1 \\
\hline Ensure nothing is put on wound site after cleaning it & 17.8 & 22.1 \\
\hline Apply plaster bandage to wound site & 7.9 & 11.9 \\
\hline
\end{tabular}

*p-value $\leq 0.05 ;$ ** p-value $\leq 0.01$

${ }^{\dagger}$ Comparisons used for PPMVs in all states except Oyo due to respondent ID error 


\section{Client characteristics at first injection and contraceptive use dynamics over} time

A total of 561 clients were contacted by telephone and surveyed within five days of receiving their first injection from a trained PPMV. Table 7 presents client characteristics at the time of their first injection. The median age of these clients was 30 years (data not shown), with more than half between the ages of 25 and 39. Most had at least some schooling (94\%), and more than half $(70 \%)$ had a secondary education or higher. Most clients were married or living with a partner $(92 \%)$ and had at least one child (94\%). One-third (33\%) did not want to have any additional children, while slightly more than one-quarter $(28 \%)$ wanted to wait at least two years before having their next child. Sixty percent of clients were employed. Forty percent of respondents had gone to the same PPMV for injectable services at least once before this visit (data not shown).

Figure 23 presents the outcome of clients' first visit to a trained PPMV for injectable contraceptive services. More than half had been using a contraceptive method before this visit: 40 percent continued with the injectable method and 19 percent switched from a different contraceptive method. Forty-two percent of clients began a new episode of use. $^{10}$ Of the 40 percent $(n=224)$ who continued using the injectable method, 65 percent received DMPA-IM, 18 percent received NET-EN, and 15 percent received DMPA-SC. Two percent did not know which method they received.

Clients were asked why they chose PPMVs for injectable contraceptive services rather than going elsewhere (Figure 24). While clients listed multiple reasons, convenient location was the most commonly cited reason $(61 \%)$. The next most commonly named reasons included anonymous care (32\%), knowing the PPMV personally (32\%), and no waiting time (31\%).

At each follow-up survey, clients who continued using an injectable contraceptive were asked whether they had experienced side effects in the three months preceding

\footnotetext{
${ }^{10}$ Clients who began a new episode of use is defined as a client who was not using a contraceptive method the day before their first visit to a trained PPMV.
}

TABLE 7. CLIENT CHARACTERISTICS AT FIRST INJECTION $(\mathrm{N}=561)^{\dagger}$

\begin{tabular}{|c|c|}
\hline & Percentage \\
\hline \multicolumn{2}{|l|}{ Age } \\
\hline $15-19$ & 1.2 \\
\hline $20-24$ & 11.1 \\
\hline $25-29$ & 31.4 \\
\hline $30-34$ & 22.3 \\
\hline $35-39$ & 21.4 \\
\hline $40-44$ & 9.1 \\
\hline $45-49$ & 3.6 \\
\hline \multicolumn{2}{|l|}{ Education } \\
\hline No formal education & 6.1 \\
\hline Primary or Koranic school & 23.5 \\
\hline Secondary school (WAEC/GCE) & 46.2 \\
\hline Higher & 24.2 \\
\hline \multicolumn{2}{|l|}{ Marital Status } \\
\hline Never married & 5.0 \\
\hline Married or in-union & 92.3 \\
\hline Widowed/divorced/separated & 2.7 \\
\hline \multicolumn{2}{|l|}{ Number of living children } \\
\hline Zero & 5.5 \\
\hline One & 11.2 \\
\hline Two & 23.0 \\
\hline Three & 21.2 \\
\hline Four & 19.4 \\
\hline Five or more & 19.3 \\
\hline \multicolumn{2}{|c|}{ How many additional children do you want to have? } \\
\hline Zero & 33.2 \\
\hline One & 14.4 \\
\hline Two & 16.6 \\
\hline Three & 12.8 \\
\hline Four or more & 18.5 \\
\hline \multicolumn{2}{|c|}{ When would you like to have your next child? } \\
\hline In the next year & 11.9 \\
\hline In the next two years & 21.0 \\
\hline More than two years from now & 28.0 \\
\hline Does not want any more children & 32.6 \\
\hline Doesn't know & 5.2 \\
\hline \multicolumn{2}{|c|}{$\begin{array}{l}\text { Had previously visited the same PPMV for injectable } \\
\text { services }\end{array}$} \\
\hline Yes & 39.8 \\
\hline No & 61.2 \\
\hline \multicolumn{2}{|l|}{ Currently employed } \\
\hline Yes & 60.4 \\
\hline No & 39.6 \\
\hline Total & 100 \\
\hline
\end{tabular}

${ }^{\dagger}$ Percentages may not add to $100 \%$ due to missing data 
the survey. At the time of their second injection, one in five clients $(20 \%)$ experienced side effects in the preceding three months $(\mathrm{n}=382)$. By the third injection, only 12 percent of clients had experienced side effects $(n=327$, data not shown).

Clients who had experienced side effects were asked whether they reported those side effects to a PPMV or to another provider (Figure 25). At each time point, over 70 percent of clients said that they reported the side effect to the PPMV. The percentage who reported a side effect to a health care provider increased from six percent to 16 percent between the second and third injection. Twenty-eight percent of clients at their second interview and 24 percent at their third interview did not report their side effects to a PPMV or other health care provider.
FIGURE 23. OUTCOME OF CLIENT'S FIRST VISIT TO A TRAINED PPMV FOR INJECTABLE SERVICES ( $N=561)$

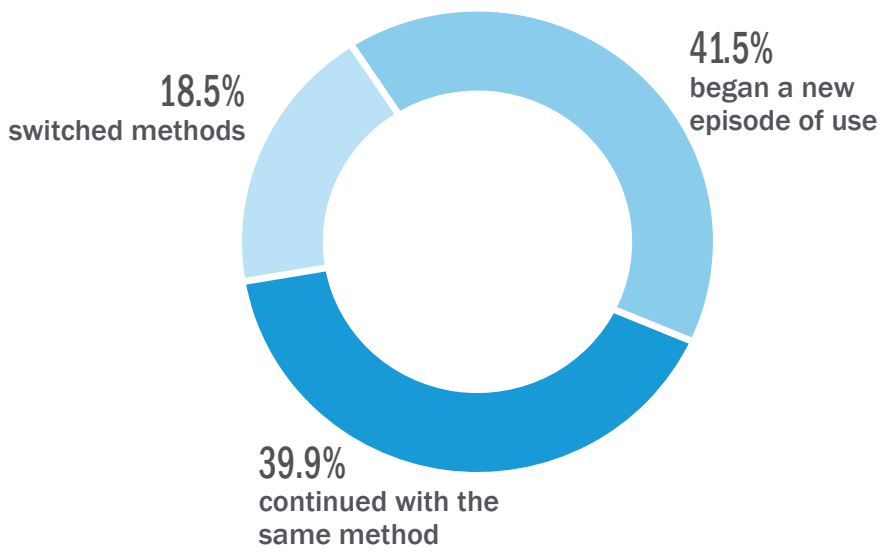

FIGURE 24. CLIENTS' REASONS FOR CHOOSING PPMVS FOR INJECTABLE CONTRACEPTIVE SERVICES AT FIRST INJECTION BY A TRAINED PPMV $(\mathrm{N}=561)^{\dagger}$

$100 \%$

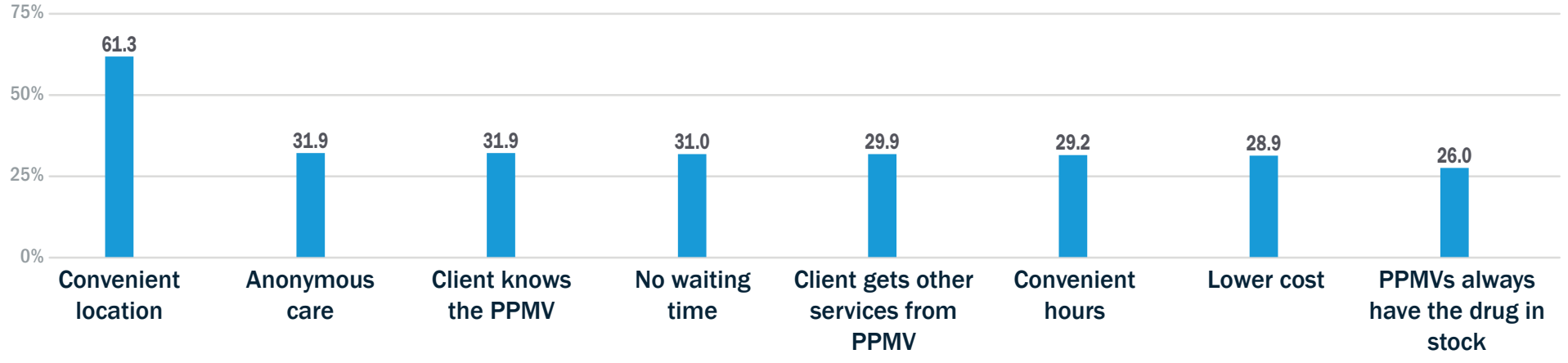

${ }^{\dagger}$ Multiple responses possible

FIGURE 25. REPORTING OF SIDE EFFECTS TO PPMVS OR OTHER

PROVIDERS, AMONG CONTINUING INJECTABLE CLIENTS WHO

EXPERIENCED SIDE EFFECTS IN THE PRECEDING THREE MONTHS

$100 \%$

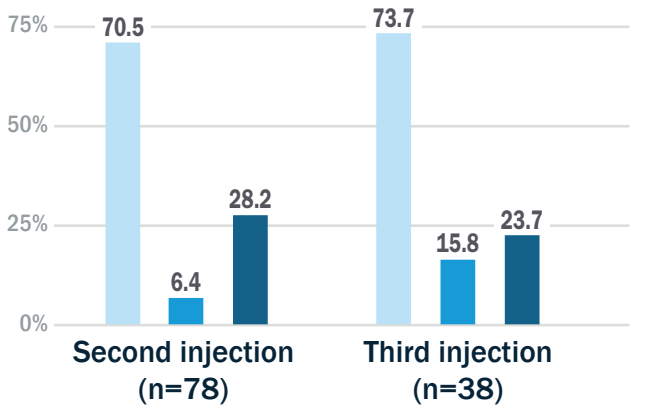

Reported side effects to PPMV

Reported side effects to other provider

Did not report side effects to provider 


\section{Clients reported receiving quality family planning counseling and were satisfied with injectable contraceptive services received from PPMVs}

To examine the quality of care received from PPMVs, clients were asked whether they received specific counseling information at each injection. Quality of care results are presented in Figure 26. Over 90 percent of clients reported receiving information on 8 of 9 counseling items at the first injection visit. Fewer clients $(82 \%)$ were told about what actions to take to resolve a side effect.

Figure 27 presents quality of care received by clients at their second and third injections. High quality of care continued among clients who returned to PPMVs for their second and third injections. Reported information received was high for all items at both the second and third injection. Over 90 percent of clients were told when to return for follow-up, felt their information would be kept confidential, felt comfortable asking questions, were told what to do if they have problems with the injectable, and were told about the potential side effects of the injectable.

FIGURE 26. QUALITY OF CARE RECEIVED AT FIRST INJECTION BY TRAINED PPMVS $(\mathrm{N}=561)$

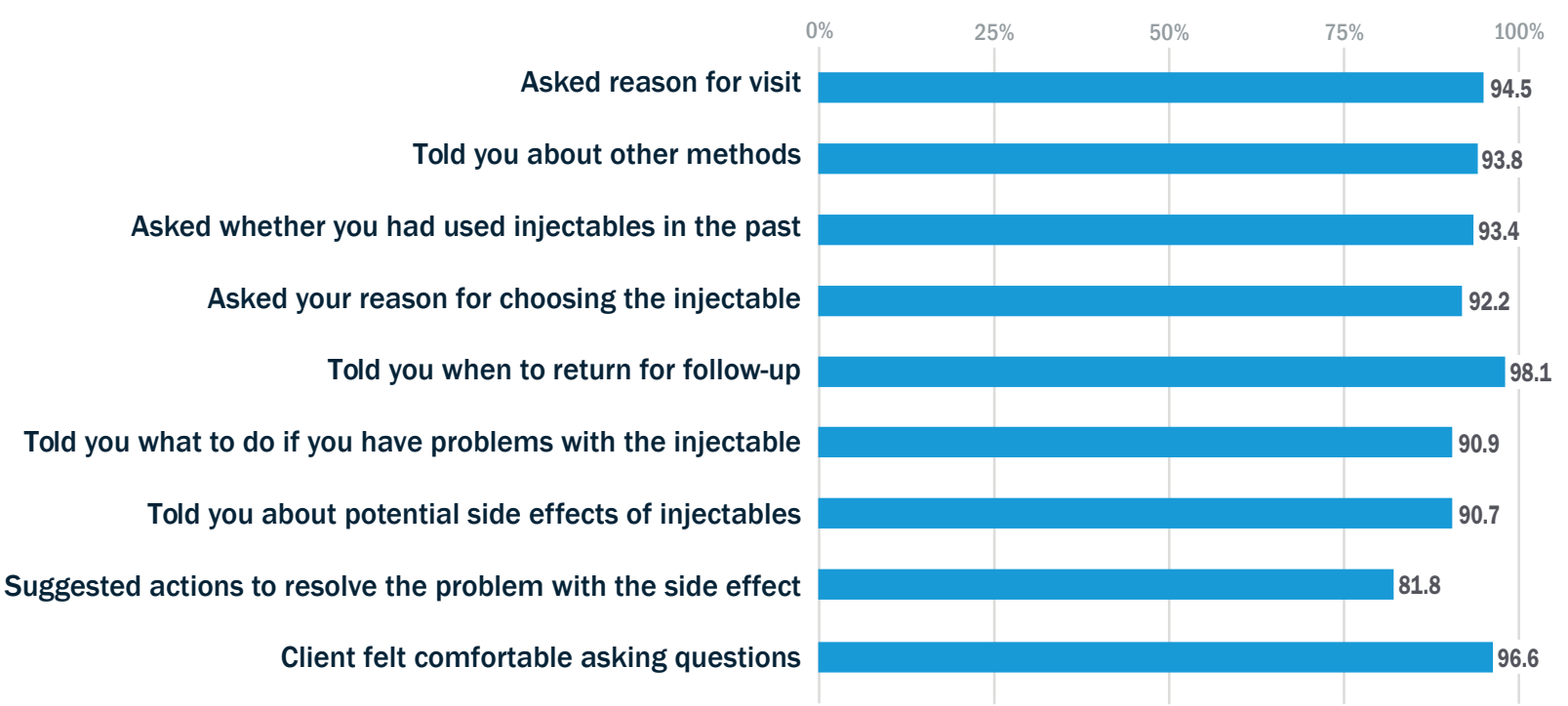

FIGURE 27. QUALITY OF CARE RECEIVED BY CONTINUING CLIENTS AT FOLLOW-UP INJECTIONS BY TRAINED PPMVS

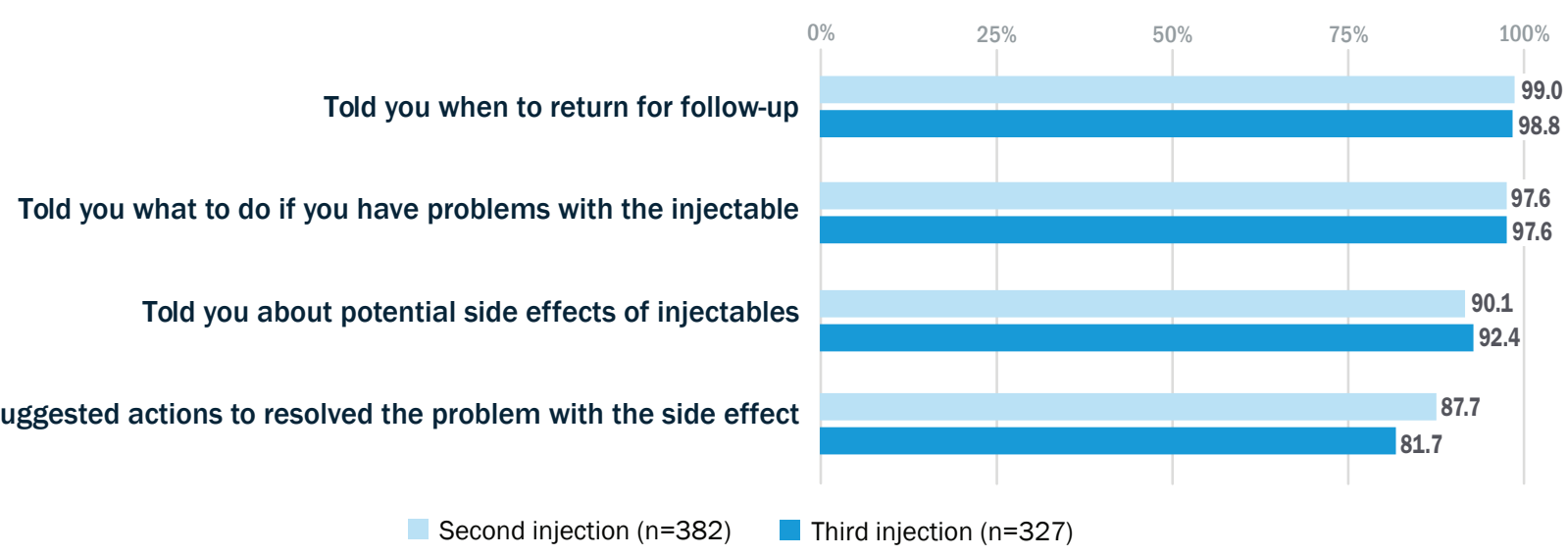


Comparing responses from Figure 26 and 27, clients reported high quality of care from the first visit, which continued to subsequent visits. Though fewer clients were given suggestions to resolve problems due to side effects, nearly 90 percent of respondents received this information at their second injection.

Figure 28 presents additional aspects of clients' experiences receiving injectable services from PPMVs. Across all survey times, nearly all respondents reported they would recommend the PPMV to a friend and that they themselves would return for injectable services. Clients in the additional four states were asked whether they found injectable contraceptive services from PPMVs affordable. Seventy-two percent of PPMVs in the additional four states felt their injectable services were affordable at FIGURE 28. OTHER ASPECTS OF CONTINUING CLIENTS' VISITS
TO TRAINED PPMVS FOR INJECTABLE CONTRACEPTIVE SERVICES

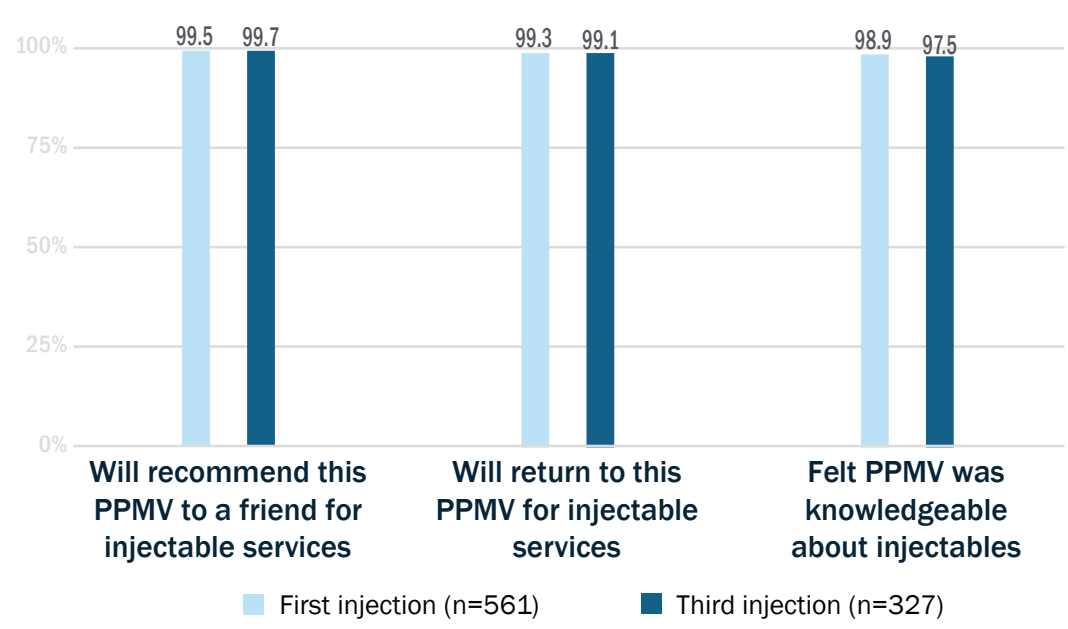
their first injection. This increased to nearly 90 percent at the second injection (data not shown).

\section{Clients who discontinued the injectable contraceptive did so for reasons unrelated to PPMV service quality}

Based on lessons learned in Nasarawa and Oyo, clients in Bauchi, Cross River, Ebonyi and Kaduna who discontinued the injectable or had not received their most recent injection were asked why they had not gone back or stopped using the method (Table 8). At the time of their second injection, 18 percent of respondents were lost to follow-up ( $n=52), 57$ percent continued using their same injectable method $(n=169), 24$ percent had discontinued the method or not received their most recent injection on time $(\mathrm{n}=71)$, and one percent $(n=2)$ had switched to another method (pill and female condoms). Among the 26 percent ( $n=73$ ) who had discontinued, had not received their most injection or switched methods, the top three reasons why clients stopped using the injectable before receiving their second injection were: not having time to go back for their injection (26\%); side effects (19\%); and their partner (12\%).

At the time of the third injection, 27 percent were lost to follow-up ( $\mathrm{n}=78$ ), 51 percent had continued their injectable method ( $n=150), 20$ percent had discontinued their injectable method $(n=60)$, and 2 percent $(n=6)$ had switched to another method (pill, implant, or condom). Among those who stopped using the injectable or PPMV injectable services before their scheduled third injection $(n=66)$, the top three reasons were: didn't have time to go back (21\%); wanted to become pregnant $(20 \%)$, and side effects $(18 \%)$. 


\section{TABLE 8. REASONS FOR STOPPING INJECTABLE METHOD AT SECOND AND THIRD}

INTERVIEW ${ }^{\dagger}$

\begin{tabular}{l|c|c}
\hline & $\begin{array}{c}\text { Second Interview } \\
(\mathbf{n = 7 3})\end{array}$ & $\begin{array}{c}\text { Third Interview } \\
(\mathbf{n = 6 6})\end{array}$ \\
\hline Didn't have time to go back for most recent injection & 26.0 & 21.2 \\
Side effects & 19.2 & 18.2 \\
Wanted to become pregnant & 12.3 & 19.7 \\
Stopped method because of partner & 12.3 & 13.6 \\
Partner or client was traveling & 9.6 & 1.5 \\
Client was pregnant & 5.5 & 9.1 \\
Lack of money & 6.8 & 0.0 \\
Forgot to go back & 5.5 & 0.0 \\
Didn't like the method/wanted to stop & 0.0 & 10.6 \\
Other & 2.7 & 6.1 \\
\hline
\end{tabular}

${ }^{\dagger}$ Only asked of clients in Bauchi, Cross River, Ebonyi and Kaduna 


\section{Discussion}

The results of this study show that many PPMVs were providing injectable contraceptives to clients who were voluntarily seeking these services from them, which is consistent with findings from previous studies (Ajuwon et al., 2013; NPC and ICF, 2014). Results also show that it is feasible for PPMVs to administer injectable contraceptive services with training, mentorship and support. The study also demonstrated that the majority of clients were satisfied with the services they received from PPMVs.

\section{PPMVs can safely provide injectable contraceptives}

After receiving a training, almost all PPMVs demonstrated the key steps for administering injectable contraceptives and this skill was retained over time. At the 6-month monitoring visit, for example, most PPMVs demonstrated all the key steps for safe DMPA-IM and DMPA-SC administration in front of a certified FP Master Trainer. Fewer PPMVs (56\%) demonstrated aspirate needle when observed at the 6-month monitoring visit. When asked to name the DMPA-IM administration steps in the 9-month follow-up survey, however, 85 percent named this step (data not shown). One reason why aspirate needle was low during the observations could have been because it is difficult to demonstrate this without a real client. Results from the survey, however, suggest that many PPMVs knew this was a key DMPA-IM administration step. Still, this DMPA-IM step should be emphasized in future trainings to ensure PPMVs understand its importance and do it routinely.

Almost all PPMVs in Bauchi, Cross River, Ebonyi and Kaduna (observation data were not collected in Oyo and Nasarawa) were observed having the sharps disposal box in their shops, suggesting that PPMVs will dispose of sharp waste safely when provided with disposal boxes. Even though PPMVs in this study were linked to local government headquarters with incineration facilities for proper disposal, the study did not assess how consistently PPMVs were taking the sharps boxes to the incineration sites. Future programmatic and research activities may look to explore barriers to PPMVs consistent disposal of sharps at incineration locations.

\section{There was no difference in knowledge and skills between PPMVs with and without previous health facility experience}

Some argue that PPMVs who previously worked in a health facility would be better equipped to provide injectable contraceptive services to clients. After the training, however, PPMVs without previous health facility experience demonstrated the same knowledge (and higher knowledge for two indicators) for 44 of the 50 indicators at the post-test survey and for 46 of the 50 indicators at the 9-month follow-up survey. Furthermore, PPMVs without health facility experience demonstrated all the same steps required for safe administration of DMPA-IM and DMPA-SC at the post-test and 6-month monitoring visit. These results strongly suggest that PPMVs without previous health facility experience perform as well as PPMVs with health facility experience when provided with proper training. Considering that only 28 percent of PPMVs in our study had previously worked in a health facility, permitting all PPMVs to provide injectable contraceptives (given they can read and write) could greatly improve access to FP and injectable services to communities across Nigeria.

\section{PPMVs' knowledge of injectable contraceptive characteristics was high after the training and was retained nine months after the training}

Although most PPMVs were already providing some type of injectable contraceptive service, PPMVs' knowledge of injectable contraceptive methods was low before the training. Across all six states, PPMVs' knowledge 
of injectable contraceptive characteristics increased immediately after the training and was retained nine months later. This suggests that PPMVs can learn from a standardized training that offers information about injectable characteristics and skills to administer injectable services. PPMVs' learned and retained some knowledge better than others. For example, less than a quarter identified either delayed return to fertility (23\%), temporary skin irritation $(21 \%)$ or decrease in sex drive $(18 \%)$ as common injectable side effects at the post-test survey. Similarly, few PPMVs named breastfeeding (21\%), rheumatic disease (19\%), migraines with aura (19\%), or deep vein thrombosis $(13 \%)$ as exclusionary health conditions at the post-test survey. These questions were based on spontaneous responses, so recall of certain side effects and exclusionary health conditions may be better than others. In this study, PPMVs were given several job aids: the MEC wheel, BCS+, and the FHI 360 screening checklist. In Bauchi, Cross River, Ebonyi and Kaduna (data not collected from PPMVs in Nasarawa and Oyo) 86 percent of PPMVs reported that they used one or more of these job aids with their clients. This suggests that the data collection methodology used in this study may not have accurately capture PPMVs knowledge and behaviors vis-à -vis what they tell clients. Most clients reported being told about side effects, which may reflect the PPMVs' use of the job aids. This also suggests that it is important to consider how these areas of knowledge can be improved: is it linked to number of clients seen or should it be emphasized in future trainings? These areas need to be better explored in future implementation activities.

\section{Clients reported receiving quality family planning counseling and were satisfied with injectable contraceptive services received from PPMVs}

Of the 561 clients interviewed, 42 percent were not using a method on the day before receiving their first injection from a trained PPMV (although some may have had previous episodes of using contraception). This suggests that PPMVs have the potential to broaden contraceptive availability to new contraceptive users. Results from the client surveys complement findings from the PPMV surveys and demonstrations that suggest PPMVs can learn and apply FP counseling and injectable contraceptive knowledge with proper support. Over 80 percent of clients reported receiving information on 11 quality of care indicators and were satisfied with injectable services received. The clients also felt that PPMVs were knowledgeable about injectable contraceptives and PPMVs gave them an opportunity to ask questions. Consequently, almost all clients were willing to return to the same PPMV for future injectable services and would refer others to the PPMV for injectable services.

\section{Clients who discontinued the injectable contraceptive did so for reasons unrelated to PPMV service quality}

Among clients who stopped using the injectable, most did so because of their desire to become pregnant. Of the few women who experienced side effects, almost all discussed the side effects they experienced with their PPMV. These findings suggest that women are willing to return to a PPMV to discuss issues that they may face with the injectable contraceptive method.

\section{Strengths \& limitations of the study}

There were several limitations of this study. First, it would have been useful to implement surveys to healthcare providers in the public sector to see how PPMVs knowledge compared to staff who are currently offering these services. Second, there were some challenges in reaching clients over time in Nasarawa and Oyo. Some clients had moved outside of the study sites or did not have steady access to a telephone/cell phone. In Bauchi, Cross River, Ebonyi, and Kaduna states, owning a phone or confirming regular access to a phone was added to the client eligibility criteria, however similar difficulties were encountered. 
Third, while there exists some demand for injectable contraceptive services by PPMVs, the average number of clients received per month was too low to include observations of client-provider interactions. Demand may also impact PPMVs knowledge and skills. Future interventions should include a demand generation component.

Fourth, PPMVs record keeping was low. Fifty percent of PPMVs observed in Bauchi, Cross River, Ebonyi and Kaduna had completed the record form provided by the study team at the 6-month monitoring visit. Record keeping is important because accurate data collection would contribute to Nigeria's ability to track contraceptive use. During the monitoring visits, many PPMVs cited police harassment for why they did not keep up-todate records. Future interventions should work with regulatory and law enforcement bodies to ensure they are aware of PPMVs' role in the delivery of injectable contraceptive services. Additional research is also needed on how to effectively motivate PPMVs to keep records.

There are many strengths to this study:

1. It was a systematic study that demonstrated PPMVs across all six geopolitical zones can provide both intramuscular and sub-cutaneous forms of the injectable contraceptive

2. Stakeholders were an integral part of the study's implementation which underpins research utilization. For example, the Bauchi commissioner of health has expressed her dedication in scaling-up PPMV provision of injectable services in her state.

\section{Study implications and recommendations}

Under the proper legal and regulatory framework, PPMVs have the potential to improve access to FP services. Results from Bauchi, Cross River, Ebonyi, Kaduna, Nasarawa and Oyo suggest that PPMVs can provide injectable contraceptives safely with proper training and support. Results from the PPMV and client data confirm that there is a demand for injectable contraceptive services from PPMVs. More importantly, clients who received services from PPMVs reported high quality of care and were satisfied with the services received.

After the training, PPMVs, regardless of their previous experience working in the formal health sector, demonstrated (a) the steps for safe administration, (b) that they could adhere to needle safety best practices, and (c) that they could gain the key knowledge to provide injectable services. While knowledge was lower for some indicators, specifically some of the side effects and exclusionary health conditions, most PPMVs reported using job aids to counsel and screen clients.

With input from various stakeholders, the training curriculum was revised based on learnings from Nasarawa and Oyo and is now ready to be used in all states for scale-up if PPMVs are authorized to provide injectable contraceptive services. Based on these findings, the Evidence Project makes the following recommendations if policy change allows for PPMV provision of injectable contraceptive services:

- All PPMVs should be trained using existing and validated materials. Most PPMVs in the additional four states strongly agreed that the curriculum used in this study improved their knowledge and skills to provide FP counseling and injectable contraceptive services.

- PPMVs should be provided job aids to for FP counseling and to counsel and screen injectable contraceptive clients.

- Linkages between the PPMVs and the public sector are needed to ensure proper referrals for any contraceptive method and disposal of sharps. 
- PPMVs require monitoring support by the state and local governments. Additional support would be needed to ensure PPMVs do not experience harassment from law officials. This potential for harassment can prohibit PPMVs from keeping up-to-date records of FP counseling, and sale and administration of injectable contraceptive services. 


\section{References}

1. Adetunji JA (1991) "Response of parents to five killer diseases among children in a Yoruba community, Nigeria.” Social Science Medicine 32:1379-1387.

2. Ajayi OO (2009). "Contraceptive knowledge and compliance with guidelines for providing contraceptive services by patent medicine vendors in Ibadan North Local Government Area." An MPH dissertation of the University of Ibadan.

3. Ajuwon AJ et al. (2013). "Research and recommendation of injectable contraceptives by patent medicine vendors in rural Nigeria." Report of a research sponsored by the WHO, Geneva, Switzerland.

4. Ajuwon AJ et al. (2006). "Outcome of interventions to improve quality reproductive health services provided by private health facilities in selected states in Nigeria." African Journal of Medicine \& Medical sciences; 35: 369-374

5. Akol A et al. (2014): “Getting closer to people: family planning provision by drug shops in Uganda." Global Health: Science and Practice 2 (4): 472-481

6. Awodele O, Adeniran A and Awodele DF. (2012). "Pharmacovigilance among patent medicine vendors in Ekiti state, Nigeria." International Journal of Risk and Safety in Medicine, 24: 65-72

7. Boniface A et al. (2006): "Community-based Study of Contraceptive Behaviour in Nigeria." African Journal of Reproductive Health / La Revue Africaine de la Sante Reproductive, Vol. 10, No. 2 pp. 90 - 104. http://www. jstor.org/stable/30032462. Accessed on 26/05/2009 13:40

8. Brieger WR (2003). "The role of patent medicine vendors in the management of sick children in the African region.” A report submitted to Basic Support for Institutionalizing Child Survival (BASICS 11)

9. Brieger WR et al. (2002): "Promoting prepackaged drugs for prompt and appropriate treatment of febrile illnesses in rural Nigerian communities." International Quarterly of Community Health Education, 21 (1): 19-40

10. Brieger WR et al. (2004) "Observations of interactions between patent medicine vendors and customers in urban and rural Nigeria." Health Policy and Planning, 19 (177-182)

11. Brugha R, Zwi A (2002) "Improving the quality of private sector delivering of public health services: challenges and strategies.” Health Policy Plan, 13:103-120.

12. Beyeler N, Liu J, Sieverding M (2015) A Systematic Review of the Role of Proprietary and Patent Medicine Vendors in Healthcare Provision in Nigeria. PLOS ONE 10(1): e0117165. doi: 10.1371/journal.pone.0117165

13. Caldwell J.C., Orubuloye I. and Caldwell P. (1992): "Fertility decline in Africa". Population and Development Review 18:2.

14. Federal Ministry of Health (2009). National Family Planning/Reproductive Health Service Protocols, Revised Edition, Nigeria.

15. Federal Ministry of Health (2004). Job description for family planning service providers, Abuja, Nigeria 
16. Fayemi M et al. (2010). "The knowledge of emergency contraception and dispensing practices of Patent Medicine Vendors in South West Nigeria." Journal of Public Health Policy, (31): 3: 281-294

17. Hoke T et al. (2012). "Community-based distribution of injectable contraceptives in four Sub-Saharan countries.” International Perspectives of Reproductive Health 38 (4): 281-294

${ }^{18 .}$ Lebetkin E et al. (2014). "Injectable contraceptive sale at licensed chemical seller shops in Ghana: access and reported use in rural and peri-urban communities." International Perspectives on Sexual and Reproductive Health 40 (1): $21-27$

19. National Bureau of Statistics (NBS) and United Nations Children's Fund (UNICEF). 2017 Multiple Indicator Cluster Survey 2016-17, Survey Findings Report. Abuja, Nigeria: National Bureau of Statistics and United Nations Children's Fund.

20. National Population Commission (2003). National Demographic Health Survey, Abuja Nigeria.

21. National Population Commission (2008). Demographic Health Survey. Abuja/Nigeria

22. National Population Commission and ICF International (2014). Nigeria Demographic and Health Survey 2013. Abuja, Nigeria and Rockville, Maryland, USA.

23. Nigerian Pharmacy Law, Chap 92, 1964. Federal Ministry of Health, Lagos, Nigeria

24. Oshiname F O. and Brieger W.R. (1992): "Primary Care Training for Patent Medicine Vendors in Rural Nigeria." Social Science and Medicine Vol. 35, No. 12, pages 1477-1484.

25. Oye-Adeniran BA et al. (2005). "Sources of contraceptive commodities for users in Nigeria." PLoS Medicine 2 (11): e306

26. Program for Appropriate Technology for Health (2014): Sayana ${ }^{\circledR}$ Press Clinical Brief. www.path.org

27. Program for Appropriate Technology for Health (2014). Frequently Asked Questions about Sayana ${ }^{\circledR}$ Press. www.path.org

28. Population Reference Bureau (2018). World Population Data Sheet. Available at: https://www.prb. org/2018-world-population-data-sheet-with-focus-on-changing-age-structures /

29. Soremekun R, Ogunbanjo O, Ogbo P.U (2013). "Accessibility of Antimalarials in Secondary Health Care Facilities and Community Pharmacies in Lagos State - A Comparative Study.” Journal of Community Medicine \& Primary Health Care 25 (2):19-27

30. World Health Organization (2007): Task shifting to tackle health worker shortages. Geneva, Switzerland

31. World Health Organization (2010): WHO best practices for injections and related procedures toolkit. Geneva, Switzerland 


\section{The Evidence Project}

Population Council

4301 Connecticut Avenue, NW, Suite 280

Washington, DC 20008 USA

tel +1 2022379400 